\title{
Article
}

\section{Coexistence of magnetic order and valence fluctuations in the Kondo lattice system Ce2Rh3Sn5}

Gamza, Monika, Gumeniuk, Roman, Burkhardt, Ulrich, Schnelle, Walter, Rosner, Helge, Leithe-Jasper, Andreas and Ślebarski, Andrzej

Available at https://clok.uclan.ac.uk/17674/

Gamza, Monika orcid iconORCID: 0000-0003-3360-4006, Gumeniuk, Roman, Burkhardt, Ulrich, Schnelle, Walter, Rosner, Helge, Leithe-Jasper, Andreas and Ślebarski, Andrzej (2017) Coexistence of magnetic order and valence fluctuations in the Kondo lattice system Ce2Rh3Sn5. Physical Review B, 95 (165142). pp. 1-12. ISSN 2469-9950

It is advisable to refer to the publisher's version if you intend to cite from the work. http://dx.doi.org/10.1103/PhysRevB.95.165142

For more information about UCLan's research in this area go to

http://www.uclan.ac.uk/researchgroups/ and search for <name of research Group>.

For information about Research generally at UCLan please go to http://www.uclan.ac.uk/research/

All outputs in CLoK are protected by Intellectual Property Rights law, including Copyright law. Copyright, IPR and Moral Rights for the works on this site are retained by the individual authors and/or other copyright owners. Terms and conditions for use of this material are defined in the policies page. 


\title{
Coexistence of magnetic order and valence fluctuations in the Kondo lattice system $\mathrm{Ce}_{2} \mathrm{Rh}_{3} \mathrm{Sn}_{5}$
}

\author{
M. B. Gamża, ${ }^{1,2,3,{ }^{*}}$ R. Gumeniuk, ${ }^{2,4}$ U. Burkhardt, ${ }^{2}$ W. Schnelle, ${ }^{2}$ H. Rosner, ${ }^{2}$ A. Leithe-Jasper, ${ }^{2}$ and A. Ślebarski ${ }^{3,5}$ \\ ${ }^{1}$ Jeremiah Horrocks Institute for Mathematics, Physics and Astrophysics, University of Central Lancashire, \\ Preston PR1 2HE, United Kingdom \\ ${ }^{2}$ Max-Planck Institute for Chemical Physics of Solids, D-01187 Dresden, Germany \\ ${ }^{3}$ Institute of Physics, University of Silesia, 40-007 Katowice, Poland \\ ${ }^{4}$ Institute of Experimental Physics, TU Bergakademie Freiberg, 09596 Freiberg, Germany \\ ${ }^{5}$ Centre for Advanced Materials and Smart Structures, Polish Academy of Sciences, 50-950 Wroctaw, Poland
}

(Received 12 December 2016; revised manuscript received 5 March 2017; published 27 April 2017)

\begin{abstract}
We report on the electronic band structure, structural, magnetic, and thermal properties of $\mathrm{Ce}_{2} \mathrm{Rh}_{3} \mathrm{Sn}_{5} . \mathrm{Ce}$ $L_{\mathrm{III}}$-edge XAS spectra give direct evidence for an intermediate valence behavior. Thermodynamic measurements reveal magnetic transitions at $T_{\mathrm{N} 1} \approx 2.9 \mathrm{~K}$ and $T_{\mathrm{N} 2} \approx 2.4 \mathrm{~K}$. Electrical resistivity shows behavior typical for the Kondo lattices. The coexistence of magnetic order and valence fluctuations in a Kondo lattice system we attribute to a peculiar crystal structure in which Ce ions occupy two distinct lattice sites. Analysis of the structural features of $\mathrm{Ce}_{2} \mathrm{Rh}_{3} \mathrm{Sn}_{5}$, together with results of electronic band structure calculations and thermodynamic and spectroscopic data indicate that at low temperatures only $\mathrm{Ce}$ ions from the $\mathrm{Ce} 1$ sublattice adopt a stable trivalent electronic configuration and show local magnetic moments that give rise to the magnetic ordering. By contrast, our study suggests that $\mathrm{Ce} 2$ ions exhibit a nonmagnetic Kondo-singlet ground state. Furthermore, the valence of $\mathrm{Ce} 2$ ions estimated from the Ce $L_{\mathrm{III}}$-edge XAS spectra varies between +3.18 at $6 \mathrm{~K}$ and +3.08 at room temperature. Thus our joined experimental and theoretical investigations classify $\mathrm{Ce}_{2} \mathrm{Rh}_{3} \mathrm{Sn}_{5}$ as a multivalent charge-ordered system.
\end{abstract}

DOI: 10.1103/PhysRevB.95.165142

\section{INTRODUCTION}

In Ce-based Kondo lattice systems, a delicate interplay of localized and itinerant electronic degrees of freedom leads to a wealth of intriguing strongly correlated electron phenomena [1-6]. Kondo lattice compounds contain Ce ions arranged periodically in a crystal lattice. Thus local $f$ moments of the $\mathrm{Ce}^{3+}$ ions can mutually couple via the conduction electrons by means of the Ruderman-Kittel-Kasayu-Yosida (RKKY) interaction [7]. Simultaneously, the $f$ moments are screened by spins of conduction $s$ electrons. This antiferromagnetic (AFM) Kondo coupling drives the demagnetization of the $f$-electron states and leads to the formation of the AbrikosovSuhl resonance near the Fermi level [1,2].

Both the RKKY interaction and Kondo effect depend on the coupling constant $J_{s-f}$ of the local $f$ moments with the conduction electron states, with the characteristic temperatures $T_{\mathrm{RKKY}} \sim J_{s-f}^{2}$ and $T_{\mathrm{K}} \sim \exp \left(1 /\left|J_{s-f}\right|\right)$, respectively [8]. In the weak-coupling limit, the magnetic interaction dominates over the Kondo spin compensation and a magnetic ground-state results [1-3]. For medium $J_{s-f}$ values, a strong competition between Kondo effect and magnetic interactions gives rise to diverse intriguing physical phenomena, including magnetism with reduced moments [3], non-Fermi liquid behavior [5] or magnetically driven superconductivity [3,4]. In turn, in the strong coupling limit, the Kondo effect predominates and leads to a nonmagnetic heavy-fermion (HF) ground state [1]. Importantly, for large $J_{s-f}$ values, the strong hybridization between the $4 f$ and other conduction-band states together with a proximity of the $4 f$ level to the Fermi energy may also trigger instabilities of the charge configuration of the $\mathrm{Ce}$

*MGamza@uclan.ac.uk ions, resulting in an intermediate valence (IV) behavior [6]. Thus physical properties of IV systems are governed by both spin fluctuations due to Kondo effect and charge fluctuations between $4 f^{0}$ and $4 f^{1}$ configurations that are nearly degenerate in energy.

While vast majority of Ce-based intermetallic compounds contains either trivalent or intermediate-valent $\mathrm{Ce}$ ions, only for a limited number of systems a coexistence of both species has been reported. Examples of such materials include $\mathrm{Ce}_{2} \mathrm{RuZn}_{4}[9,10], \mathrm{Ce}_{3} \mathrm{Ni}_{2} \mathrm{Ge}_{7}[11], \mathrm{Ce}_{23} \mathrm{Ru}_{7} X_{4}(X=\mathrm{Mg}, \mathrm{Cd})$ [12,13], CeRuSn [14-16], $\mathrm{Ce}_{5} \mathrm{Sn}_{3}$ [17], $\mathrm{Ce}_{7} T_{3}$ (T-transition metal) [18], and $\mathrm{Ce}_{4-x} \mathrm{Ru}_{4} \mathrm{Ge}_{12+x}$ [19]. These systems often exhibit remarkable electronic and magnetic properties related to a mixture of Ce ions with long and extraordinarily short distances to the neighboring atoms due to a peculiar bonding situation. Bearing this distinctive structural feature in mind, we started systematic investigations aiming at finding novel intermetallic compounds with highly unconventional magnetic behavior resulting from the presence of $\mathrm{Ce}$ ions in different valence states.

We focused our search on the system Ce-Rh-Sn as it is rich in ternary phases that show a full spectrum of strongly correlated electron phenomena related to various strength of hybridization between $\mathrm{Ce} 4 f$ and other valence-band states [20-27]. $\mathrm{CeRhSn} 2, \mathrm{Ce}_{5} \mathrm{Rh}_{4} \mathrm{Sn}_{10}, \mathrm{CeRh}_{2} \mathrm{Sn}_{4}$, and $\mathrm{Ce}_{3} \mathrm{Rh}_{4} \mathrm{Sn}_{13}$ are magnetically ordered Kondo lattice systems [20-23]. In contrast, for $\mathrm{Ce}_{3+x} \mathrm{Rh}_{4} \mathrm{Sn}_{13-x}(0.2 \lesssim x \lesssim 0.6)$, no sign of Kondo effect or long range magnetic order was found even down to $0.4 \mathrm{~K}$ [25]. In turn, in CeRhSn the Ce ions are in an IV state [26,27].

Here, we report on $\mathrm{Ce}_{2} \mathrm{Rh}_{3} \mathrm{Sn}_{5}$ that crystallizes in the orthorhombic $\mathrm{Y}_{2} \mathrm{Rh}_{3} \mathrm{Sn}_{5}$ type of structure, where $\mathrm{Y}$ ions occupy two distinct crystallographic sites [28,29]. An early study revealed a moderate $\mathrm{HF}$ behavior $\left(\gamma \approx 150 \mathrm{~mJ} \mathrm{Ce} \mathrm{mol}^{-1} \mathrm{~K}^{-2}\right)$ 
with a magnetic transition at $T_{N} \approx 5 \mathrm{~K}$ (from resistivity), $4 \mathrm{~K}$ (from magnetic susceptibility), or $2.5 \mathrm{~K}$ (from heat capacity) [28]. Our single-crystal $\mathrm{X}$-ray diffraction (XRD) study unveils extraordinarily short $\mathrm{Ce}-\mathrm{Rh}$ contacts indicative of valence larger than $3+$ for one $\mathrm{Ce}$ site. Interestingly, the local environment of $\mathrm{Ce}$ atoms from the second sublattice is very similar to that in $\mathrm{CeRh}_{2} \mathrm{Sn}_{4}$, a magnetic Kondo lattice system with trivalent $\mathrm{Ce}$ ions and $T_{N} \approx 3.2 \mathrm{~K}$ [23]. Motivated by these results, we performed a combined experimental and theoretical study on $\mathrm{Ce}_{2} \mathrm{Rh}_{3} \mathrm{Sn}_{5}$ based on thermodynamic measurements and spectroscopic data together with firstprinciples electronic structure calculations aiming at exploring its complex structural and magnetic properties.

\section{METHODS}

\section{A. Experimental}

Polycrystalline samples of $\mathrm{Ce}_{2} \mathrm{Rh}_{3} \mathrm{Sn}_{5}$ with total weight of about $2 \mathrm{~g}$ were prepared from ingots of cerium (Ames, 99.9 wt.\%), rhodium granules (ChemPur, 99.9 wt.\%), and tin foil (ChemPur, 99.995 wt.\%). Stoichiometric amounts of the elemental metals were arc-melted on a water cooled copper hearth in an ultrahigh purity Ar atmosphere with a $\mathrm{Zr}$ getter (heated above the melting point). The sample was remelted several times to promote homogeneity and heat-treated at $800{ }^{\circ} \mathrm{C}$ for 14 days in a sealed Ta tube enclosed in an evacuated quartz tube. Almost no mass loss (below 0.02\%) occurred during the melting and annealing processes.

All manipulations related to preparation of the sample and its storage were performed in a argon-filled glove box [MBRAUN, $\mathrm{p}\left(\mathrm{O}_{2} / \mathrm{H}_{2} \mathrm{O}\right) \leqslant 1 \mathrm{ppm}$ ] in order to prevent the oxidation. The quality of the sample was examined by means of powder XRD measurements and metallographic investigations. Details of these studies are included in Ref. [30]. Powder XRD pattern indicates that the sample is nearly single phased. Microprobe measurements revealed the chemical composition that corresponds to $\mathrm{Ce}_{2.01(1)} \mathrm{Rh}_{3.00(2)} \mathrm{Sn}_{5.00(2)}$ and thus confirms the desired stoichiometry.

An irregularly shaped crystal was mechanically extracted from the annealed ingot. A single-crystal XRD study was performed at room temperature using an Xcalibur E Single Crystal Diffractometer. Details concerning data collection and handling are summarized in Table I. Structure refinements were carried out using the JANA2006 program [33].

The Ce $L_{\text {III }}$ XAS spectra were recorded in transmission arrangement at the EXAFS beamline $\mathrm{C}$ of HASYLAB at DESY at the temperatures of 80 and $293 \mathrm{~K}$. The wavelength selection was realized using the four-crystal mode, which yielded an experimental resolution of $\sim 2 \mathrm{eV}$ (FWHM) at the Ce $L_{\text {III }}$ threshold of $5723 \mathrm{eV}$. Powdered samples of $\mathrm{Ce}_{2} \mathrm{Rh}_{3} \mathrm{Sn}_{5}$ were mixed with small amounts of $\mathrm{B}_{4} \mathrm{C}$ and mounted on $1 \mathrm{~cm}^{2}$ window sample holders using paraffin wax. Two series of measurements performed using different sample powders at ambient temperature and at low temperatures down to $6 \mathrm{~K}$ using a helium gas flow cryostat gave consistent results. Experimental data were recorded with $\mathrm{CePO}_{4}$ as the external reference compound with $\mathrm{Ce}^{3+}$ ions. The Ce $L_{\mathrm{III}}$ XAS spectra were evaluated using the ATHENA program package [34].
TABLE I. Crystallographic data for $\mathrm{Ce}_{2} \mathrm{Rh}_{3} \mathrm{Sn}_{5}$.

\begin{tabular}{|c|c|}
\hline Empirical formula & $\mathrm{Ce}_{2} \mathrm{Rh}_{3} \mathrm{Sn}_{5}$ \\
\hline Structure type & $\mathrm{Y}_{2} \mathrm{Rh}_{3} \mathrm{Sn}_{5}$ \\
\hline Space group & $C m c 2_{1}$ (No. 36) \\
\hline Lattice parameters ${ }^{\mathrm{a}}$ & $\begin{array}{l}a=4.4992(1) \AA \\
b=26.4839(7) \AA \\
c=7.2160(2) \AA\end{array}$ \\
\hline Unit cell volume, ${ }^{a} V$ & $859.83(4) \AA^{3}$ \\
\hline Formula units/cell, $Z$ & 4 \\
\hline Crystal density, $\rho$ & $9.151 \mathrm{~g} \mathrm{~cm}^{-3}$ \\
\hline Temperature & $295(5) \mathrm{K}$ \\
\hline Diffraction system & $\begin{array}{l}\text { Xcalibur E, four-circle Kappa } \\
\text { Sapphire CCD Detector (Xcalibur) }\end{array}$ \\
\hline Radiation, $\lambda$ & Mo $K \alpha, 0.71073 \AA$ \\
\hline Range in $h, k, l$ & $\pm 9, \pm 52, \pm 14$ \\
\hline$R(e q v) / R(\sigma)$ & $0.078 / 0.018$ \\
\hline $2 \theta_{\min } / 2 \theta_{\max }$ & $4.17 / 45.49$ \\
\hline Observation criteria & $F(h k l)>3.00 \sigma(F)$ \\
\hline Resolution $d(\AA)$ & 0.45 \\
\hline Absorption correction & face-based, analytical [31] \\
\hline Absorption coefficient & 30.194 \\
\hline$N(h k l)$ measured & 71975 \\
\hline$N(h k l)$ unique & 3939 \\
\hline Extinction method & isotropic type 2 correction [32] \\
\hline Extinction coefficient & $4070(70)$ \\
\hline Number of parameters & 63 \\
\hline Goodness of fit (GOF) & 1.94 \\
\hline$R$ & $2.25 \%$ \\
\hline$w R$ & $3.28 \%$ \\
\hline
\end{tabular}

${ }^{\text {a Powder data. }}$

XPS experiments were performed at room temperature using a PHI 5700 ESCA spectrometer with monochromatized $\mathrm{Al} \mathrm{K}_{\alpha}$ radiation. The energy resolution was about $0.4 \mathrm{eV}$. The polycrystalline sample was broken under a high vacuum of $6 \times 10^{-10}$ Torr immediately before measuring the spectra. Binding energies were referenced to the Fermi level $\left(E_{\mathrm{F}}=0\right)$. Calibration of the spectra was performed according to Ref. [35].

The magnetization studies were carried out in a SQUID magnetometer (MPMS XL-7, Quantum Design) at temperatures between 1.8 and $400 \mathrm{~K}$ in magnetic fields up to $70 \mathrm{kOe}$. Electrical resistivity measurements were performed with a standard dc four-probe setup. Heat capacity was determined by a relaxation-type method using a commercial system (PPMS, Quantum Design).

\section{B. Computational}

The electronic structure of $\mathrm{Ce}_{2} \mathrm{Rh}_{3} \mathrm{Sn}_{5}$ was studied using the full potential local orbital (FPLO) minimum basis code [36]. Scalar-relativistic calculations based on the density functional theory (DFT) were performed within the local (spin) density approximation [L(S)DA] using the Perdew and Wang [37] parametrization of the exchange-correlation $(\mathrm{XC})$ potential. The strong Coulomb correlation within the Ce $4 f$ shell was also treated in a mean field approximation using the $\mathrm{LSDA}+U$ method [38] with the around mean field double counting scheme. The Coulomb repulsion $U$ and exchange constant $J$ for the $4 f$ states of both types of Ce atoms were assumed to 
TABLE II. Atomic positional and displacement parameters for $\mathrm{Ce}_{2} \mathrm{Rh}_{3} \mathrm{Sn}_{5}$ (Note: $B_{12}=B_{23}=0$.) All atoms are located at $4 a(0, y, z)$ Wyckoff positions. The experimental structural data are compared to those derived from the band structure calculations in the LDA approximation. The free parameters in atomic coordinates obtained from the computational study were rounded to three significant digits.

\begin{tabular}{|c|c|c|c|c|c|c|c|c|c|}
\hline \multirow[b]{2}{*}{ Atom } & \multicolumn{7}{|c|}{ experiment } & \multicolumn{2}{|c|}{ LDA } \\
\hline & $y$ & $z$ & $B_{11}$ & $B_{22}$ & $B_{33}$ & $B_{23}$ & $B_{\text {iso }}$ & $y$ & $z$ \\
\hline $\mathrm{Ce} 1$ & $0.32754(1)$ & $0.27397(5)$ & $0.72(1)$ & $0.77(1)$ & $0.98(1)$ & $-0.02(1)$ & $0.82(1)$ & 0.330 & 0.274 \\
\hline $\mathrm{Ce} 2$ & $0.02426(1)$ & $0.25076(4)$ & $0.93(1)$ & $0.77(1)$ & $0.81(1)$ & $-0.08(1)$ & $0.84(1)$ & 0.026 & 0.239 \\
\hline Rh1 & $0.72296(2)$ & $0.28274(6)$ & $0.75(1)$ & $0.74(1)$ & $0.87(2)$ & $-0.06(1)$ & $0.79(1)$ & 0.723 & 0.280 \\
\hline $\mathrm{Rh} 2$ & $0.10594(2)$ & $0.00000(6)$ & $1.06(2)$ & $0.82(1)$ & $0.94(2)$ & $0.01(1)$ & $0.94(1)$ & 0.103 & 0.999 \\
\hline $\mathrm{Rh} 3$ & $0.44897(2)$ & $0.03180(6)$ & $0.78(1)$ & $0.82(1)$ & $0.80(2)$ & $0.00(1)$ & $0.80(1)$ & 0.450 & 0.015 \\
\hline Sn1 & $0.21037(1)$ & $0.49104(5)$ & $0.80(1)$ & $0.82(1)$ & $0.73(1)$ & $-0.07(1)$ & $0.78(1)$ & 0.210 & 0.491 \\
\hline $\operatorname{Sn} 2$ & $0.20563(1)$ & $0.07177(6)$ & $0.71(1)$ & $0.82(1)$ & $0.79(1)$ & $0.09(1)$ & $0.77(1)$ & 0.205 & 0.065 \\
\hline $\mathrm{Sn} 3$ & $0.62182(1)$ & $0.25218(6)$ & $1.41(1)$ & $0.69(1)$ & $0.93(1)$ & $-0.09(1)$ & 1.01(1) & 0.622 & 0.242 \\
\hline $\mathrm{Sn} 4$ & $0.45116(1)$ & $0.41543(6)$ & $0.86(1)$ & $0.73(1)$ & $0.77(1)$ & $0.01(1)$ & $0.79(1)$ & 0.452 & 0.400 \\
\hline Sn5 & $0.09635(1)$ & $0.62444(6)$ & $0.77(1)$ & $0.76(1)$ & $0.99(1)$ & $0.16(1)$ & $0.84(1)$ & 0.096 & 0.618 \\
\hline
\end{tabular}

be $1-8$ and $1 \mathrm{eV}$, respectively. Thus the effective $U_{\text {eff }}=U-J$ was from the range of $0-7 \mathrm{eV}$. The Brillouin zone sampling was based on $198 \mathbf{k}$ points in the irreducible wedge (2000 points in the full zone). A series of calculations with an increasing density of the $\mathbf{k}$ mesh was performed to ensure the convergence of the total energy with respect to the $\mathbf{k}$ grid.

Based on the band structure results we estimated the theoretical XPS valence-band spectra. The partial $l$-resolved densities of states obtained using the LDA and the LSDA $+U$ ( $U=6 \mathrm{eV}, J=1 \mathrm{eV}$ ) methods were weighted by their respective photoionisation cross-sections [39]. The results were multiplied by the Fermi-Dirac function for $300 \mathrm{~K}$ and convoluted by Lorentzians with a full width at half maximum (FWHM) of $0.4 \mathrm{eV}$ to account for the instrumental resolution, thermal broadening and the lifetime effect of the hole states.

\section{RESULTS AND DISCUSSION}

\section{A. Crystal structure}

Refinement of the single crystal XRD data shows that $\mathrm{Ce}_{2} \mathrm{Rh}_{3} \mathrm{Sn}_{5}$ crystallizes with the noncentrosymmetric orthorhombic $\mathrm{Y}_{2} \mathrm{Rh}_{3} \mathrm{Sn}_{5}$ type of structure (space group $C m c 2_{1}$ ), in agreement with earlier reports [28,29]. The crystallographic details of the refinement are given in Table I, atomic coordinates and anisotropic displacement parameters in Table II, and interatomic distances in Table III.

The $\mathrm{Y}_{2} \mathrm{Rh}_{3} \mathrm{Sn}_{5}$ type is a layered structure consisting of two analogical layers shifted by $1 / 2$ of the translation in the $b c$-plane and alternating along $x$ direction. As indicated in Fig. 1, each layer consists of two main fragments: A (a chain of interconnected $\mathrm{Y} 2 / \mathrm{Ce} 2$ atoms and edge sharing tetragons and triangles) and $\mathrm{B}$ (condensed pentagons and octagons). The octagons are centered by heavy Y1/Ce1 atoms, while pentagons remains empty. The B fragments are very similar to layers formed by octagons and pentagons in the structure of the $\mathrm{NdRh}_{2} \mathrm{Sn}_{4}$ type. The close structural relationship of $\mathrm{Y}_{2} \mathrm{Rh}_{3} \mathrm{Sn}_{5}$ and $\mathrm{NdRh}_{2} \mathrm{Sn}_{4}$ types is widely discussed in the literature $[23,29,40]$.

The interatomic distances in the structure of $\mathrm{Ce}_{2} \mathrm{Rh}_{3} \mathrm{Sn}_{5}$ (Table III) mostly correlate well with the sum of atomic radii of the elements $\left[r_{(\mathrm{Ce})}=1.825 \AA ; r_{(\mathrm{Rh})}=1.34 \AA\right.$; and $r_{(\mathrm{Sn})}=1.41 \AA$ ] [41]. The Sn-Sn contacts are slightly longer than $2.81 \AA$, while the $\mathrm{Rh}$-Sn distances are shortened by about $4 \%-5 \%$, which assumes the formation of covalently bonded Rh-Sn framework in the investigated structure. The shrinking of $\mathrm{Ce}-\mathrm{Sn}$ contacts is of $1 \%-2 \%$, assuming also a weak interaction.

TABLE III. Selected interatomic distances in $\mathrm{Ce}_{2} \mathrm{Rh}_{3} \mathrm{Sn}_{5}$.

\begin{tabular}{|c|c|c|c|c|c|}
\hline Atoms & & $d(\AA)$ & Atoms & & $d(\AA)$ \\
\hline \multirow[t]{7}{*}{ Ce1 - } & $2 \mathrm{Sn} 1$ & $3.1979(4)$ & & $1 \mathrm{Ce} 1$ & $3.4733(5)$ \\
\hline & $2 \mathrm{Sn} 5$ & $3.2060(4)$ & & $1 \mathrm{Sn} 2$ & $3.0255(5)$ \\
\hline & $2 \mathrm{Sn} 2$ & $3.2310(4)$ & & $1 \mathrm{Sn} 5$ & $3.1670(5)$ \\
\hline & $1 \mathrm{Sn} 4$ & $3.4268(5)$ & & $2 \mathrm{Sn} 2$ & $3.2155(4)$ \\
\hline & $1 \mathrm{Sn} 1$ & $3.4733(5)$ & & 2Rh1 & $2.7250(3)$ \\
\hline & $1 \mathrm{Sn} 2$ & $3.5402(5)$ & & 1Rh1 & $2.7452(6)$ \\
\hline & 2Rh2 & $3.2885(4)$ & $\mathrm{Sn} 2-$ & $2 \mathrm{Ce} 1$ & $3.2310(4)$ \\
\hline \multirow[t]{7}{*}{$\mathrm{Ce} 2-$} & $2 \mathrm{Sn} 4$ & $3.1954(3)$ & & $1 \mathrm{Ce} 1$ & $3.5402(5)$ \\
\hline & $1 \mathrm{Sn} 5$ & $3.3012(5)$ & & $1 \mathrm{Sn} 1$ & $3.0255(5)$ \\
\hline & $1 \mathrm{Sn} 5$ & $3.3190(5)$ & & $2 \mathrm{Sn} 1$ & $3.2155(4)$ \\
\hline & $2 \mathrm{Sn} 4$ & $3.3656(4)$ & & $1 \mathrm{Rh} 2$ & $2.6885(6)$ \\
\hline & $2 \mathrm{Sn} 3$ & $3.4243(4)$ & & 2Rh1 & $2.7537(4)$ \\
\hline & 1Rh2 & $2.8180(5)$ & & 1Rh1 & $2.8132(6)$ \\
\hline & 2Rh3 & $3.1091(4)$ & Sn3 - & $2 \mathrm{Ce} 2$ & $3.4243(4)$ \\
\hline \multirow[t]{5}{*}{ Rh1 - } & $1 \mathrm{Sn} 3$ & $2.6854(5)$ & & $1 \mathrm{Sn} 4$ & $3.1024(5)$ \\
\hline & $2 \mathrm{Sn} 1$ & $2.7250(3)$ & & 1Rh1 & $2.6854(5)$ \\
\hline & $1 \mathrm{Sn} 1$ & $2.7452(6)$ & & $1 \mathrm{Rh} 3$ & $2.7522(6)$ \\
\hline & $2 \mathrm{Sn} 2$ & $2.7537(4)$ & & $2 \mathrm{Rh} 2$ & $2.9226(4)$ \\
\hline & $1 \mathrm{Sn} 2$ & $2.8132(6)$ & Sn4 - & $2 \mathrm{Ce} 2$ & $3.1954(3)$ \\
\hline \multirow[t]{6}{*}{ Rh2 - } & $1 \mathrm{Ce} 2$ & $2.8180(5)$ & & $2 \mathrm{Ce} 2$ & $3.3656(4)$ \\
\hline & $2 \mathrm{Ce} 1$ & $3.2885(4)$ & & $1 \mathrm{Ce} 1$ & $3.4268(5)$ \\
\hline & $1 \mathrm{Sn} 2$ & $2.6885(6)$ & & $1 \mathrm{Sn} 3$ & $3.1024(5)$ \\
\hline & $1 \mathrm{Sn} 5$ & $2.7196(6)$ & & 1Rh3 & $2.7665(6)$ \\
\hline & $2 \mathrm{Sn} 4$ & $2.7774(3)$ & & $1 \mathrm{Rh} 3$ & $2.7729(6)$ \\
\hline & $2 \mathrm{Sn} 3$ & $2.9226(4)$ & & $2 \mathrm{Rh} 2$ & $2.7774(3)$ \\
\hline \multirow[t]{5}{*}{ Rh3 - } & $2 \mathrm{Ce} 2$ & $3.1091(4)$ & Sn5 - & $2 \mathrm{Ce} 1$ & $3.2060(4)$ \\
\hline & $2 \operatorname{Sn} 5$ & $2.6350(3)$ & & $1 \mathrm{Ce} 2$ & $3.3012(5)$ \\
\hline & $1 \mathrm{Sn} 3$ & $2.7522(6)$ & & $1 \mathrm{Ce} 2$ & $3.3190(5)$ \\
\hline & $1 \mathrm{Sn} 4$ & $2.7665(6)$ & & $1 \mathrm{Sn} 1$ & $3.1670(5)$ \\
\hline & $1 \mathrm{Sn} 4$ & $2.7729(6)$ & & $2 \mathrm{Rh} 3$ & $2.6350(3)$ \\
\hline Sn1 - & $2 \mathrm{Ce} 1$ & $3.1979(4)$ & & $1 \mathrm{Rh} 2$ & $2.7196(6)$ \\
\hline
\end{tabular}




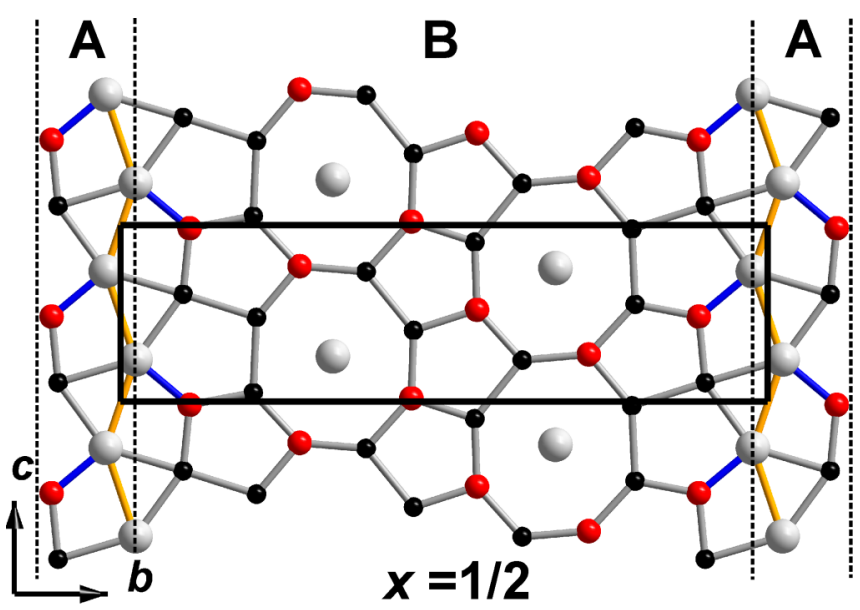

FIG. 1. The planar layer in the structure of $\mathrm{Ce}_{2} \mathrm{Rh}_{3} \mathrm{Sn}_{5}$ occurring at $x=1 / 2$. Fragment A: a chain of interconnected Ce atoms (yellow line) and edge sharing tetragons and triangles; fragment $\mathrm{B}$ : condensed empty pentagons and octagons of $\mathrm{Rh}$ (red) and $\mathrm{Sn}$ (black) centered by Ce (grey) atoms.

The most intriguing feature of the $\mathrm{Ce}_{2} \mathrm{Rh}_{3} \mathrm{Sn}_{5}$ structure is the shortened $\mathrm{Ce} 2-\mathrm{Rh} 2$ distance by nearly $11 \%$ as compared to the sum of atomic radii of $\mathrm{Ce}$ and $\mathrm{Rh}$. Such extraordinarily short $\mathrm{Ce} 2-\mathrm{Rh}$ contacts indicate that the valence of $\mathrm{Ce} 2$ ions should be larger than $+3[6,27,42,43]$. In turn, for $\mathrm{Ce} 1$ ions, all the Ce1-Sn and Ce1-Rh distances are nearly equal to the sum of the corresponding atomic radii. This suggests that the electronic configuration for $\mathrm{Ce} 1$ ions is close to $4 f^{1}$, i.e., $\mathrm{Ce}^{+3}$. Thus the analysis of the crystal structure indicates that $\mathrm{Ce}_{2} \mathrm{Rh}_{3} \mathrm{Sn}_{5}$ may be a mixed valence, IV or even a multivalent charge-ordered system. The last scenario assumes a static ordering of trivalent and intermediate-valent $\mathrm{Ce}$ ions in two distinct lattice sites and was proposed for systems such as $\mathrm{Ce}_{2} \mathrm{RuZn}_{4}[9,10], \mathrm{Ce}_{3} \mathrm{Ni}_{2} \mathrm{Ge}_{7}[11], \mathrm{Ce}_{23} \mathrm{Ru}_{7} X_{4}(X=\mathrm{Mg}, \mathrm{Cd})$ $[12,13], \mathrm{Ce}_{5} \mathrm{Sn}_{3}$ [17], and $\mathrm{YbPtGe}_{2}$ [44]. To inspect the valence states of $\mathrm{Ce}$ ions in $\mathrm{Ce}_{2} \mathrm{Rh}_{3} \mathrm{Sn}_{5}$ more closely, we performed spectroscopic measurements.

\section{B. Ce $L_{\text {III }}$ XAS}

Figure 2 presents the near-edge regime of Ce $L_{\mathrm{III}} \mathrm{XAS}$ spectra for $\mathrm{Ce}_{2} \mathrm{Rh}_{3} \mathrm{Sn}_{5}$ that were recorded at several temperatures between 6 and $300 \mathrm{~K}$. Although the spectra are dominated by "a white line" at the energy of $\sim 5726 \mathrm{eV}$ corresponding to $4 f^{1}$ configuration of $\mathrm{Ce}^{3+}$, there is also an additional contribution at the energy of nearly $+9 \mathrm{eV}$ above the white line maximum that indicates the presence of $\mathrm{Ce}^{4+}$ species with electron configuration $4 f^{0}$. Increasing temperature from 6 to $300 \mathrm{~K}$ leads to a gradual reduction of the high-energy contribution due to $4 f^{0}$ configuration of $\mathrm{Ce}^{4+}$ and a simultaneous slight increase in intensity of the white line, as shown in Fig. 2(b). Such a progressive shift of the spectral weight to lower energies implies an IV behavior. Although the temperature induced changes in the Ce $L_{\mathrm{III}}$ XAS spectra are rather small, similar slight effects were reported for many Ce-based IV compounds $[6,43,45,46]$.

Deconvolution of the Ce $L_{\mathrm{III}}$ XAS spectra [an example in Fig. 2(a)] indicates that the mean Ce valence in $\mathrm{Ce}_{2} \mathrm{Rh}_{3} \mathrm{Sn}_{5}$

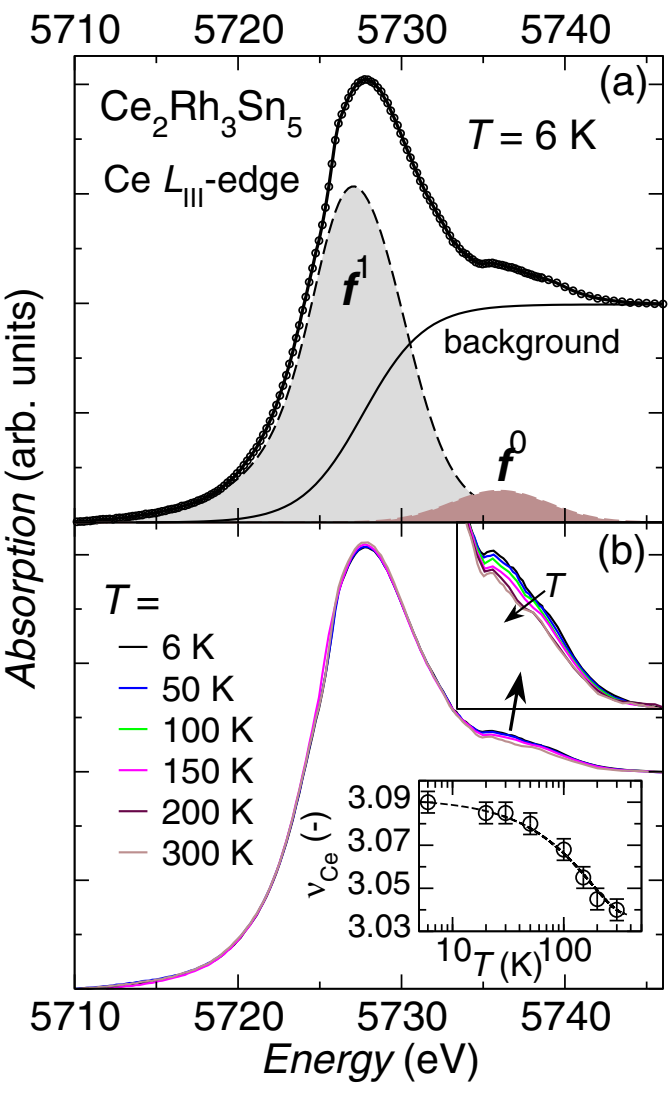

FIG. 2. Near-edge regime of Ce $L_{\mathrm{III}} \mathrm{XAS}$ spectra for $\mathrm{Ce}_{2} \mathrm{Rh}_{3} \mathrm{Sn}_{5}$. (a) shows deconvolution of the normalized spectrum recorded at $T=6 \mathrm{~K}$. The contributions due to $f^{1}$ and $f^{0}$ configurations are indicated by beige or grey fields underneath the dashed lines, while the solid black line represents the arctan function that accounts for transitions of $2 p_{3 / 2}$ electrons to the extended conduction states. (b) depicts normalized Ce $L_{\mathrm{III}}$ XAS spectra measured at various temperatures between 6 and $300 \mathrm{~K}$. The changes in intensity of the $f^{0}$ contribution are enlarged in the upper inset, with the arrow indicating the direction of increasing temperature. The lower inset of (b) displays the calculated changes of $\mathrm{Ce}$ valence as a function of temperature. The dashed line is a guide to the eye.

decreases from $+3.09(1)$ at $6 \mathrm{~K}$ to $+3.04(1)$ at ambient temperature, with the most rapid changes taking place at temperatures of $100 \mathrm{~K}$ [see lower inset in Fig. 2(b)]. However, since the measured spectra contain signal originating from all $\mathrm{Ce}$ ions, the obtained values of Ce valence in $\mathrm{Ce}_{2} \mathrm{Rh}_{3} \mathrm{Sn}_{5}$ should be interpreted as the average valence for $\mathrm{Ce}$ ions from two distinct lattice sites. Therefore, assuming that $\mathrm{Ce} 1$ ions are trivalent in the entire temperature range, the valence of $\mathrm{Ce} 2$ species should vary between +3.18 at $6 \mathrm{~K}$ and +3.08 at $\sim 300 \mathrm{~K}$. We note that similar Ce valence was reported for nonmagnetic IV compounds such as $\mathrm{CeRhSi}_{2}$ [43], $\mathrm{Ce}_{2} \mathrm{Ni}_{3} \mathrm{Si}_{5}$ [47], and $\mathrm{CeMo}_{2} \mathrm{Si}_{2} \mathrm{C}$ [48]. To shed more light on the character of Ce $4 f$ states in $\mathrm{Ce}_{2} \mathrm{Rh}_{3} \mathrm{Sn}_{5}$, we performed $\mathrm{X}$-ray photoelectron spectroscopy measurements.

\section{Ce $3 d$ XPS}

Figure 3 shows room-temperature Ce $3 d$ XPS spectrum for $\mathrm{Ce}_{2} \mathrm{Rh}_{3} \mathrm{Sn}_{5}$ together with the data for the structurally related 


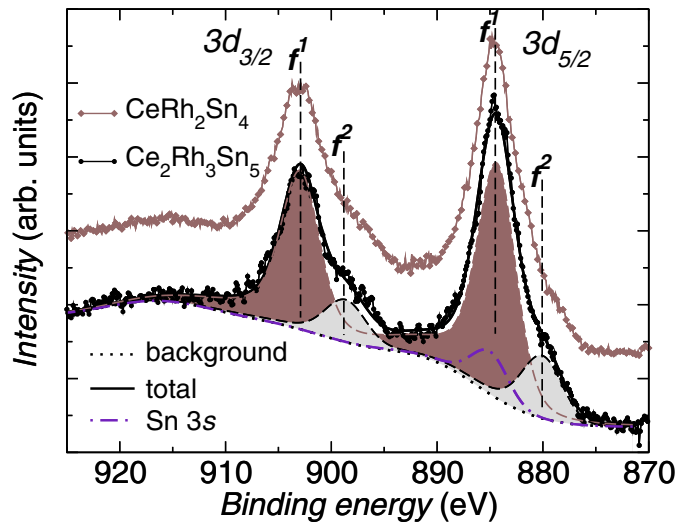

FIG. 3. The Ce $3 d$ XPS spectra for $\mathrm{Ce}_{2} \mathrm{Rh}_{3} \mathrm{Sn}_{5}$ and $\mathrm{CeRh}_{2} \mathrm{Sn}_{4}$. The spin-orbit components $3 d_{32}$ and $3 d_{52}$ as well as the $f^{1}$ and $f^{2}$ contributions are labeled. The deconvolution of the spectrum for $\mathrm{Ce}_{2} \mathrm{Rh}_{3} \mathrm{Sn}_{5}$ is shown.

compound $\mathrm{CeRh}_{2} \mathrm{Sn}_{4}$. Due to the spin-orbit (SO) coupling, $\mathrm{Ce} 3 d$ states give rise to two sets of photoemission lines that correspond to the $j=3 / 2$ and $j=5 / 2$ components of final states. Each of these SO sets consists of two distinct contributions labeled as $f^{1}$ and $f^{2}$. The $f^{1}$ components originate from the screening of the core hole by conduction electrons. The $f^{2}$ satellites arise when an electron is transferred from extended valence-band states to the $4 f$ states during photoemission process in order to screen the hole in the core shell. Since the probability of such processes depends on the coupling of the $4 f$ levels to the other states near the Fermi level [42], the presence of the pronounced $f^{2}$ peaks indicates that there is a notable hybridization between the Ce $4 f$ and conduction-band states in $\mathrm{Ce}_{2} \mathrm{Rh}_{3} \mathrm{Sn}_{5}$.

There are no additional peaks in the Ce $3 d$ XPS spectrum of $\mathrm{Ce}_{2} \mathrm{Rh}_{3} \mathrm{Sn}_{5}$ in a distance of $\sim 11 \mathrm{eV}$ from the main photoemission lines, that could be assigned to the $4 f^{0}$ final states. This result may seem to contradict the mean $\mathrm{Ce}$ valence of +3.04 at ambient temperature obtained from our XAS measurements (see Sec. III B). We note, however, XPS measurements with energy resolution of $0.4 \mathrm{eV}$ may not show weak $f^{0}$ contributions. Our simulations (not shown) indicate that only peaks with intensities greater than $\sim 5 \%$ of the combined intensity of $f^{1}$ and $f^{2}$ contributions should be detectable in the Ce $3 d$ XPS spectrum of $\mathrm{Ce}_{2} \mathrm{Rh}_{3} \mathrm{Sn}_{5}$, whereas weaker $f^{0}$ contributions should be hidden in the background signal and experimental noise. Thus the absence of $f^{0}$-type peaks implies the mean occupancy of the Ce $4 f$ shell $n_{f} \gtrsim 0.95$ at room temperature, which agrees with the results of our XAS study (see Sec. III B).

Deconvolution of the Ce $3 d$ XPS spectrum was performed on the basis of Doniach-Šunjić theory [49]. The intensity ratio $I\left(3 d_{5 / 2}\right) / I\left(3 d_{3 / 2}\right)=3 / 2$ was fixed during the fitting, as required by the quantum-mechanical rules. The SO split $\delta_{\mathrm{s}-\mathrm{O}} \approx 18.6 \mathrm{eV}$ was assumed. A Tougaard-type background [50] was subtracted from the XPS data. A small peak due to the Sn $3 s$ states located at the binding energy of $885 \mathrm{eV}$ was included in the fit, with the intensity determined by the stoichiometry of the compound $\mathrm{Ce}_{2} \mathrm{Rh}_{3} \mathrm{Sn}_{5}$. Model calculations of Gunnarsson and Schönhammer (GS) [42,51] were used to calculate the value of the $\Delta$ parameter describing the hybridization strength between the Ce $4 f$ shell and conduction electron states from relative intensities of the $f^{2}$ peaks. Such a procedure yielded $\Delta \approx 100 \mathrm{meV}$, which should be considered as the average hybridization parameter for $\mathrm{Ce}$ ions occupying two lattice sites in $\mathrm{Ce}_{2} \mathrm{Rh}_{3} \mathrm{Sn}_{5}$.

The obtained $\Delta$ value is comparable to those found in systems with $\mathrm{Ce}$ in an IV state $(\Delta \gtrsim 100 \mathrm{meV})[26,27,42]$ and is notably stronger than in other compounds from the ternary Ce-Rh-Sn system with trivalent Ce ions $(\Delta \lesssim 80 \mathrm{meV})$ [20,22-25]. In particular, the $\Delta$ value for $\mathrm{Ce}_{2} \mathrm{Rh}_{3} \mathrm{Sn}_{5}$ is larger than that for the structurally related compound $\mathrm{CeRh}_{2} \mathrm{Sn}_{4}$ with $\mathrm{Ce}^{3+}$, as evidenced directly by larger intensities of $f^{2}$ satellites in the Ce $3 d$ XPS spectra (see Fig. 3).

Despite the strong hybridization $\Delta$ and valence fluctuations revealed by our spectroscopic investigations, thermodynamic measurements indicate a magnetic ground state for $\mathrm{Ce}_{2} \mathrm{Rh}_{3} \mathrm{Sn}_{5}$.

\section{Magnetic measurements}

Figures 4 and 5 present results of a $d c$ magnetization study on polycrystals of $\mathrm{Ce}_{2} \mathrm{Rh}_{3} \mathrm{Sn}_{5}$. The $M(T)$ measured in weak magnetic fields increases rapidly at temperatures below $\sim 2.9 \mathrm{~K}$, suggestive of an onset of magnetic order [see Fig. 4(a)]. Closer inspection shows that the $M(T)$ grows even faster at $T \leqslant 2.4 \mathrm{~K}$. This points to a change in magnetic structure at $2.4 \mathrm{~K}$, in agreement with our specific heat data that revealed two anomalies at $T_{\mathrm{N} 1} \approx 2.9 \mathrm{~K}$ and $T_{\mathrm{N} 2} \approx 2.4 \mathrm{~K}$ (see Sec. IIIE). The presence of two magnetic transitions in $\mathrm{Ce}_{2} \mathrm{Rh}_{3} \mathrm{Sn}_{5}$ provides an explanation for the discrepancy between ordering temperatures reported by Patil et al. [28] based on the specific heat and magnetization data.

Isothermal magnetization curves measured at temperatures below $3 \mathrm{~K}$ show distinct hysteresis between the data collected with increasing and decreasing field strengths. The illustrative $M(H)$ data recorded at $T=1.8 \mathrm{~K}$ are shown in Fig. 4(b). Both the remanence magnetization $M_{r}$ and the coercive field decrease gradually with increasing temperature and finally diminish at $T_{\mathrm{N} 1} \approx 2.9 \mathrm{~K}$ [see the lower inset of Fig. 4(b)]. However, the magnetization in the ordered states is very small, about two orders of magnitude smaller than magnetization values expected for a ferromagnetic order that involves one magnetic ion per formula unit with $S=1 / 2$. Thus the magnetization measurements suggest an AFM ordering with only small canting in $\mathrm{Ce}_{2} \mathrm{Rh}_{3} \mathrm{Sn}_{5}$ at temperatures below $2.4 \mathrm{~K}$, with the canting becoming even smaller between 2.4 and $2.9 \mathrm{~K}$.

At high temperatures the magnetic susceptibility $\chi=M / H$ does not depend on the applied magnetic field and resembles behavior expected for a local moment paramagnet (see the inset of Fig. 4). As shown in the inset of Fig. 5, at temperatures between $\sim 170$ and $400 \mathrm{~K}$ the $\chi^{-1}(T)$ follows a modified Curie-Weiss law:

$$
\chi(T)=\frac{C_{\mathrm{HT}}}{T-\theta_{\mathrm{HT}}}+\chi_{0-\mathrm{HT}},
$$

with $\chi_{0-\mathrm{HT}} \approx 8 \times 10^{-5} \mathrm{emu} / \mathrm{mol}$, the paramagnetic Weiss temperature $\theta_{\mathrm{HT}} \approx-103 \mathrm{~K}$ and the Curie constant $C_{\mathrm{HT}}=1.60 \mathrm{emu} \mathrm{K} / \mathrm{mol}$. The obtained $C_{\mathrm{HT}}$ value corresponds to the fluctuating magnetic moment of $2.51 \mu_{\mathrm{B}}$ per $\mathrm{Ce}$, which 

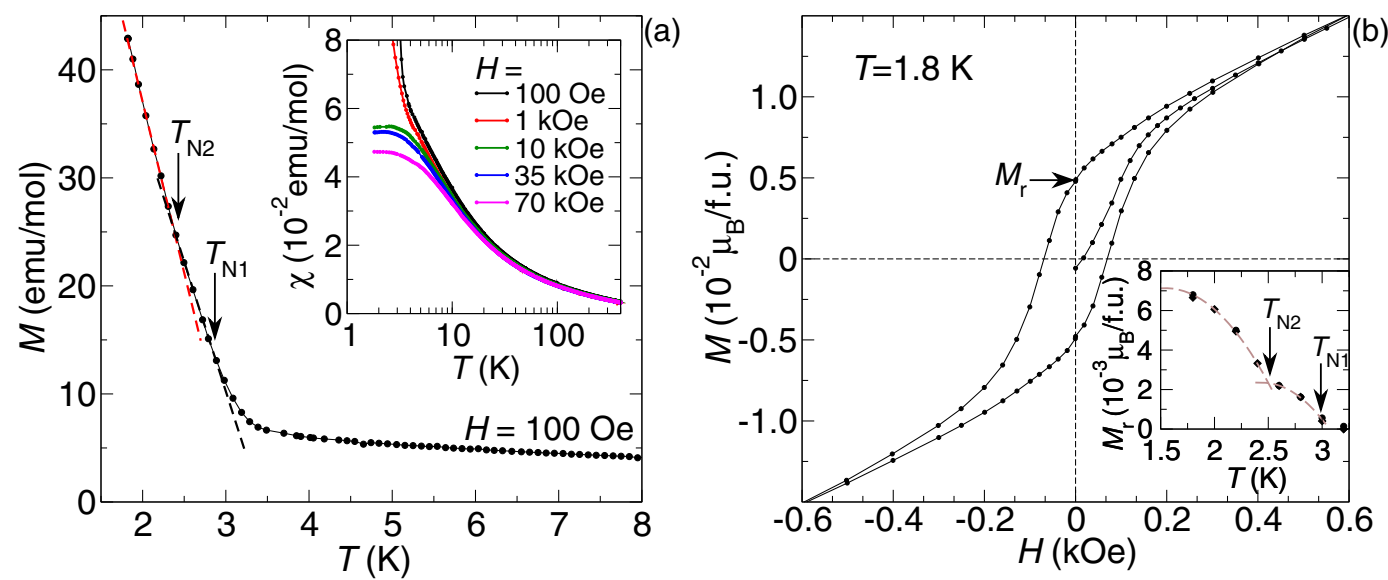

FIG. 4. Results of $d c$ magnetization measurements for $\mathrm{Ce}_{2} \mathrm{Rh}_{3} \mathrm{Sn}_{5}$. (a) shows the low-temperature part of the $M(T)$ measured in magnetic field of 100 Oe. Two distinct changes in slope of the $M(T)$ curve suggestive of magnetic transitions are indicated. The inset displays the magnetic susceptibility measured in a number of magnetic fields between 100 Oe and $70 \mathrm{kOe}$ and plotted as a function of temperature on a logarithmic scale. (b) depicts the full magnetization loop at $T=1.8 \mathrm{~K}$. Inset shows values of the remanence magnetization at various temperatures. The dashed lines are guided to the eye.

is very close to the effective moment of $2.53 \mu_{\mathrm{B}} / \mathrm{Ce}$ expected for free $\mathrm{Ce}^{3+}$ ions. Thus the magnetic measurements indicate that at temperatures above $\sim 170 \mathrm{~K}$ the valence of Ce ions in $\mathrm{Ce}_{2} \mathrm{Rh}_{3} \mathrm{Sn}_{5}$ is close to $3+$ and all the Ce ions bear localized magnetic moments.

The small positive $\chi_{0-\mathrm{HT}}$ is in-line with metallic properties of $\mathrm{Ce}_{2} \mathrm{Rh}_{3} \mathrm{Sn}_{5}$. The $\chi_{0-\mathrm{HT}}$ is the sum of the diamagnetic susceptibility of the closed-shells and the conduction electron contributions. Assuming that for tin $\chi_{\text {dia }}\left(\mathrm{Sn}^{4+}\right)=-16 \times 10^{-6} \mathrm{emu} / \mathrm{mol}$, for cerium $\chi_{\mathrm{dia}}\left(\mathrm{Ce}^{3+}\right)=$ $-20 \times 10^{-6} \mathrm{emu} / \mathrm{mol}$ and for rhodium $\chi_{\mathrm{dia}}\left(\mathrm{Rh}^{4+}\right)=$

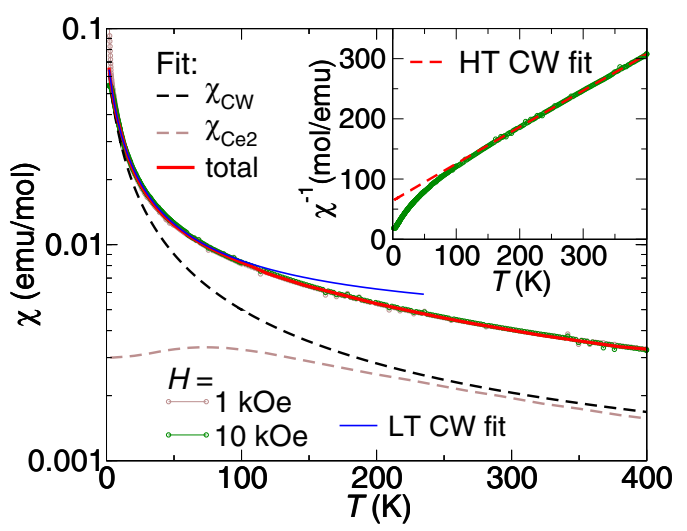

FIG. 5. The dc magnetic susceptibility of $\mathrm{Ce}_{2} \mathrm{Rh}_{3} \mathrm{Sn}_{5}$ measured in magnetic fields of $1 \mathrm{kOe}$ (brown circles) and $10 \mathrm{kOe}$ (green circles), together with the fit using Eq. (2) (thick solid red line). The Curie-Weiss-type contribution $\chi_{\mathrm{CW}}$ due to Ce1 sublattice and the $\chi_{\mathrm{Ce} 2}$ term estimated based on calculations of Rajan [54] to account for the magnetic susceptibility originating from $\mathrm{Ce} 2$ ions are indicated with black and brown dashed lines, respectively. The solid blue line represents the best fit to the $\chi(T)$ data at temperatures of 8-85 K by using the modified Curie-Weiss law. The inset shows $\chi^{-1}(T)$ in a magnetic field of $10 \mathrm{kOe}$ (green circles) together with the fit by using the modified Curie-Weiss law that covers the data above $\sim 170 \mathrm{~K}$ (dashed red line).
$-18 \times 10^{-6} \mathrm{emu} / \mathrm{mol}[52]$, the sum of the diamagnetic core increments yields $-174 \times 10^{-6} \mathrm{emu} / \mathrm{mol}$. Thus the rough estimate of the electronic Pauli susceptibility $\chi_{\mathrm{P}}$, after correcting for the Landau electron diamagnetism $\chi_{\mathrm{L}}=-\frac{1}{3} \chi_{\mathrm{P}}$ and the core-level diamagnetism, gives $381 \times 10^{-6} \mathrm{emu} / \mathrm{mol}$. This value corresponds to the $\operatorname{DOS}\left(E_{\mathrm{F}}\right) \approx 11.8$ states $\mathrm{eV}^{-1}$ f.u. ${ }^{-1}$, which is comparable to the value of the $\operatorname{DOS}\left(E_{\mathrm{F}}\right)$ obtained from our electronic band structure calculations (Fig. 9).

At lower temperatures, the experimental $\chi(T)$ data can be well described by the modified Curie-Weiss law (see Fig. 5) with a small negative Weiss temperature $\theta_{\mathrm{LT}} \approx-6 \mathrm{~K}$ of the order of $T_{\mathrm{N} 1}, \chi_{\mathrm{LT}} \approx 0.0039 \mathrm{emu} / \mathrm{mol}$ and the Curie constant $C_{\mathrm{LT}}=0.48 \mathrm{emu} \mathrm{K} / \mathrm{mol}$, which is strongly reduced as compared to the value of $1.60 \mathrm{emu} \mathrm{K} / \mathrm{mol}$ expected for full moments of $\mathrm{Ce}^{3+}$ ions. The hefty lowering of the effective fluctuating moment accompanied by a strong enhancement of the Pauli susceptibility upon lowering temperature points to the delocalization of $4 f$ electrons from some of $\mathrm{Ce}$ ions in $\mathrm{Ce}_{2} \mathrm{Rh}_{3} \mathrm{Sn}_{5}$. The large negative Weiss temperature $\theta_{\mathrm{HT}} \approx-110 \mathrm{~K}$ indicates that there is a strong AFM coupling of the local $4 f$ moments with conduction-band states. In Ce-based Kondo lattice systems, this interaction drives the demagnetization of the $f$-electron states and can lead to the formation of a nonmagnetic Kondo-singlet state, for which an enhanced Pauli-like magnetic susceptibility results from the presence of a narrow peak in the quasiparticle DOS near the Fermi energy due to the Abrikosov-Suhl resonance [6,53].

Since there are two distinct Ce sites in $\mathrm{Ce}_{2} \mathrm{Rh}_{3} \mathrm{Sn}_{5}$ (see Sec. III A), we attempted to fit the experimental magnetic susceptibility in a broad temperature range as

$$
\chi(T)=\frac{C}{T-\theta}+\chi_{\mathrm{Ce} 2}(T),
$$

with the first term describing Curie-Weiss behavior anticipated for local moments of $\mathrm{Ce}^{3+}$ ions from $\mathrm{Ce} 1$ sublattice and the second term $\chi_{\mathrm{Ce} 2}(T)$ included to account for magnetic susceptibility of $\mathrm{Ce} 2$ ions calculated based on $\chi\left(T / T_{0}\right) / \chi(0)$ curve obtained by Rajan [54] for trivalent $\mathrm{Ce}$ impurities 
$(j=5 / 2)$ embedded in a see of conduction electrons. Here, $T_{0}$ stands for a characteristic temperature that reflects the strength of coupling between the Ce $4 f$ and conduction-band states, and $\chi(0)$ denotes the Pauli-like magnetic susceptibility of a nonmagnetic Kondo-singlet state at zero temperature. A very good fit to the experimental $\chi(T)$ data at temperatures between $\sim 10$ and $400 \mathrm{~K}$ was achieved assuming $T_{0} \approx 280 \mathrm{~K}$, $\chi(0) \approx 0.003 \mathrm{emu} / \mathrm{mol}$, the paramagnetic Weiss temperature $\theta \approx-6 \mathrm{~K}$ and the Curie constant $C=0.48 \mathrm{emu} \mathrm{K}(\mathrm{mol} \mathrm{Ce})^{-1}$ (see Fig. 4).

The negative Weiss temperature of $-6 \mathrm{~K}$ is comparable to the magnetic ordering temperature and concurs with the dominance of weak AFM exchange interactions between local magnetic moments of $\mathrm{Ce}$ ions from the $\mathrm{Ce} 1$ sublattice. The estimated $C$ value corresponds to the fluctuating moment of $1.96 \mu_{\mathrm{B}}$ per $\mathrm{Ce} 1$, which is somewhat smaller than the magnetic moment of $2.54 \mu_{\mathrm{B}}$ expected for free $\mathrm{Ce}^{3+}$ ions. We note that the local environment of $\mathrm{Ce} 1$ ions in the crystal lattice of $\mathrm{Ce}_{2} \mathrm{Rh}_{3} \mathrm{Sn}_{5}$ is very similar to that in $\mathrm{CeRh}_{2} \mathrm{Sn}_{4}$ [23], an antiferromagnet with well localized $\mathrm{Ce}^{3+}$ moments. Since for $\mathrm{CeRh}_{2} \mathrm{Sn}_{4}$ the effective fluctuating moment decreases at temperatures below $\sim 150 \mathrm{~K}$ due to a thermal depopulation of excited crystal field levels from the $j=5 / 2$ multiplet of $\mathrm{Ce}^{3+}$ ions [23], we suspect that the effective moment derived from the fit of the $\chi(T)$ for $\mathrm{Ce}_{2} \mathrm{Rh}_{3} \mathrm{Sn}_{5}$ using Eq. (2) may be influenced by crystal electric field (CEF) effect on the magnetic Ce1 ions.

Since Eq. (2) does not consider CEF effects, the reliability of the performed fit is rather limited, especially regarding the detailed temperature dependence of the $\chi_{\mathrm{Ce} 2}$ term which is small as compared to the magnetic susceptibility originating from local magnetic moments of $\mathrm{Ce} 1$ ions in $\mathrm{Ce}_{2} \mathrm{Rh}_{3} \mathrm{Sn}_{5}$. In addition, Eq. (2) does not account for the influence of valence fluctuations revealed by the XAS measurements (see Sec. III B) on the magnetic susceptibility due to $\mathrm{Ce} 2$ ions in $\mathrm{Ce}_{2} \mathrm{Rh}_{3} \mathrm{Sn}_{5}$. Nevertheless, the temperature of the maximum in the $\chi_{\mathrm{Ce} 2}(T)$ derived from the fit of $\sim 80 \mathrm{~K}$ is close to the value of the ratio $C\left(\mathrm{Ce}^{3+}\right) /(3 \chi(0)) \approx 89 \mathrm{~K},\left[C\left(\mathrm{Ce}^{3+}\right)\right.$ is the Curie constant for $\left.\mathrm{Ce}^{3+}\right]$, as expected for IV systems with nearly trivalent Ce.[6] Furthermore, the maximum in the $\chi_{\mathrm{Ce} 2}(T)$ occurs in the same temperature range in which our XAS study revealed the fastest changes in Ce valence in $\mathrm{Ce}_{2} \mathrm{Rh}_{3} \mathrm{Sn}_{5}$. This finding agrees with the observation of Lawrence et al. [6], that a single energy scale describes both charge and spin excitations associated with intermediate-valent $\mathrm{Ce}$ ions in systems with Ce valence close to $3+$. Finally, it is worthwhile to note that the $\chi_{\mathrm{Ce} 2}(T)$ term obtained from the fit closely resembles the magnetic susceptibility for $\mathrm{CeRhSi}_{2}$, a nonmagnetic IV system with similar $\mathrm{Ce}$ valence, in which $\mathrm{Ce}$ ions occupy only one lattice site [43].

Based on the Coqblin-Schrieffer model for orbitally degenerate $\mathrm{Ce}^{3+}$ ions, the Kondo temperature $T_{\mathrm{K}}$ describing the rate of spin fluctuations arising from hybridization of $\mathrm{Ce}$ $4 f$ and conduction-band states is related to the characteristic temperature $T_{0}$ as follows: $T_{\mathrm{K}}=2 \pi T_{0} W(N) / N$, where the degeneracy factor $N=6(N=2 j+1)$ and the Wilson number $W(N=6)=0.6464[55,56]$. Therefore the performed analysis of the magnetic susceptibility of $\mathrm{Ce}_{2} \mathrm{Rh}_{3} \mathrm{Sn}_{5}$ gives an estimate of the Kondo temperature for $\mathrm{Ce} 2$ ions of $189 \mathrm{~K}$.
Finally, from the $\chi(T)$ data, we conclude that the lowtemperature magnetic properties of $\mathrm{Ce}_{2} \mathrm{Rh}_{3} \mathrm{Sn}_{5}$ can be explained as a superposition of the enhanced Pauli paramagnetism $[\chi(0) \approx 0.003 \mathrm{emu} / \mathrm{mol}]$ due to delocalized $4 f$ electrons of $\mathrm{Ce} 2$ ions and a magnetic order followed by local moment paramagnetic behavior resulting from the presence of well localized magnetic moments of trivalent $\mathrm{Ce} 1$ ions. To further test this scenario, we performed specific heat measurements.

\section{E. Specific heat}

Figure 6 shows the low-temperature specific heat data for $\mathrm{Ce}_{2} \mathrm{Rh}_{3} \mathrm{Sn}_{5}$. A distinct anomaly in the $C_{\mathrm{p}}(T)$ curve at $2.4 \mathrm{~K}$ and a shoulder at $2.9 \mathrm{~K}$ are consistent with results of magnetic measurements (see Sec. IIID) and indicate that $\mathrm{Ce}_{2} \mathrm{Rh}_{3} \mathrm{Sn}_{5}$ undergoes two magnetic transitions. However, the low-temperature specific heat is dominated by a broad contribution underneath the magnetic peaks. To inspect its origin, we perform quantitative analysis of different contributions to the low-temperature specific heat.

First, we evaluate the lattice and electronic specific heat. To this end, we plotted the $C_{\mathrm{p}}$ data in a $C_{\mathrm{p}}(T) / T$ representation [see Fig. 6(b)] and applied the Debye model. At temperatures between $\sim 11$ and $\sim 20 \mathrm{~K}$, the experimental data follow the dependence $C_{\mathrm{p}} / T=\gamma+\beta T^{2}+\delta T^{4}$, where the Sommerfeld coefficient $\gamma \approx 110 \mathrm{~mJ} \mathrm{~mol}^{-1} \mathrm{~K}^{-2}$ accounts for the electronic part of the specific heat and the consecutive two terms with $\beta \approx 1.66 \times 10^{-3} \mathrm{~mJ} \mathrm{~mol}^{-1} \mathrm{~K}^{-4}$ and $\delta \approx 4.04 \times 10^{-7} \mathrm{~mJ} \mathrm{~mol}^{-1} \mathrm{~K}^{-6}$ represent the first two terms in the Taylor expansion for the lattice specific heat. Although the fit was performed on the specific heat data at rather high temperatures, we note that the upper limit of the temperature range used for this analysis is only of $25 \%$ of the temperature at which $\chi_{\mathrm{Ce} 2}(T)$ shows a maximum due to spin excitations from Kondo singlet ground state to $j=5 / 2$ multiplet for nonmagnetic Ce ions (see Sec. III D). Further, the obtained $\beta$ value corresponds to the Debye temperature of $227 \mathrm{~K}$, which is close to Debye temperatures reported by Patil et al. [28] for isostructural and isoelectronic compounds with other rare earth elements.

To check whether the estimated $\gamma$ value correlates with the enhancement of the zero-temperature magnetic susceptibility $\chi(0) \approx 3 \times 10^{-3} \mathrm{emu} / \mathrm{mol}$ (see Sec. IIID) and thus could be explained as due to itinerant $f$ electrons in the FL state, we calculate the Sommerfeld-Wilson ratio $R_{\mathrm{SW}}=\left(\pi^{2} k_{\mathrm{B}}^{2} \chi(0) /\left(3 \mu_{\mathrm{eff}}^{2} \gamma\right)\right.$ using the free-ion value of the effective fluctuating moment $\mu_{\text {eff }}$ of $2.54 \mu_{\mathrm{B}}$ per $\mathrm{Ce}^{3+}$ ion. The resulting $R_{\mathrm{SW}} \approx 1$ is typical for $\mathrm{HF}$ systems in which strong hybridization between $4 f$ and conduction-band states leads to the delocalization of the $4 f$ electrons, giving rise to an enhancement of the effective mass at low temperatures [57].

The magnetic specific heat $C_{m}(T)$ was calculated by subtracting from the total $C_{p}(T)$ the estimated lattice and electronic contributions [see Fig. 6(a)]. The $C_{m}(T) / T$ was extrapolated to $T=0$ and then integrated with respect to temperature to give an estimate for the low-temperature magnetic entropy, $S_{\mathrm{m}}(T)$. As shown in the inset of Fig. 6(b), the $S_{\mathrm{m}}(T)$ saturates at the value of $0.8 \mathrm{R} \ln 2$ per formula unit. Even with the electronic specific heat term $\gamma T$ included in the 

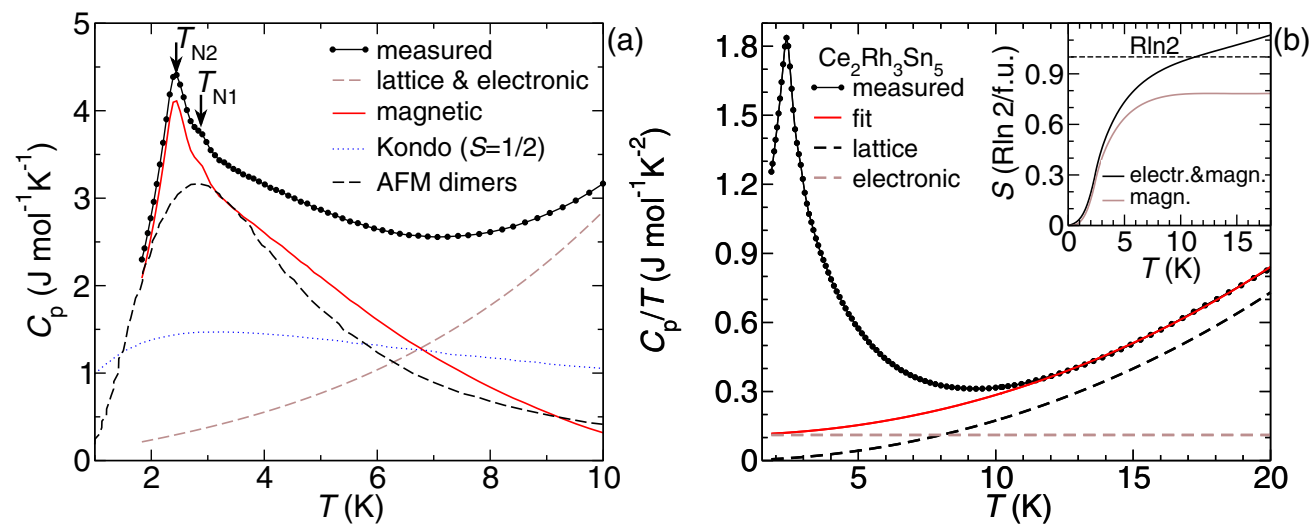

FIG. 6. Low-temperature specific heat of $\mathrm{Ce}_{2} \mathrm{Rh}_{3} \mathrm{Sn}_{5}$ shown as $C_{\mathrm{p}}(T)$ (a) and plotted in a conventional $C_{p} / T(T)$ representation (b). The experimental data (black circles) are shown together with results of fits based on the Debye model as described in the text. The magnetic contribution (red solid line) as well as fits based on the Kondo model for $S=1 / 2$ [59] (blue dots) and simulation of the magnetic specific heat of AFM dimers (black dashed line) are also presented in (a). The inset in (b) depicts the entropy per formula unit of $\mathrm{Ce}_{2} \mathrm{Rh}_{3} \mathrm{Sn}_{5}$ calculated from the magnetic specific heat (solid brown line) and based on the sum of the electronic and magnetic specific heat (black solid line). The dashed horizontal line indicates the value of $\mathrm{R} \ln 2$ expected for one Ce ion, a magnetic doublet ground state.

estimate, the magnetic entropy is only of $\mathrm{R} \ln 2$ per formula unit containing two $\mathrm{Ce}$ ions. This result implies that only half of $\mathrm{Ce}$ ions in $\mathrm{Ce}_{2} \mathrm{Rh}_{3} \mathrm{Sn}_{5}$ is in a CEF doublet ground state and contributes to the low-temperature magnetic ordering.

The magnetic entropy saturates well above the magnetic transitions only, at $T \approx 10 \mathrm{~K}$ [see inset of Fig. 6(b)]. Importantly, the value of the $S_{\mathrm{m}}$ recovered at $T_{\mathrm{N} 2}=2.9 \mathrm{~K}$ is only of $0.40 \mathrm{R} \ln 2$ per formula unit. Such a strong reduction of the magnetic entropy can be attributed to the Kondo effect on magnetic $\mathrm{Ce}$ ions and/or short range magnetic correlations that develop above magnetic ordering temperature. Assuming that the effect results solely from partial quenching of $\mathrm{Ce} 4 f$ derived magnetic moments of $\mathrm{Ce}^{3+}$ ions by Kondo effect, the single-ion Kondo temperature for magnetic Ce ions estimated based on model calculations of Yashima et al. [58] for $S=1 / 2$ Kondo impurity should be of $7 \mathrm{~K}$. However, attempts to describe the broad contribution to the magnetic specific heat based on the Kondo model [59] do not give satisfactory results [see Fig. 6(a)]. Instead, the shape of the $C_{m}(T)$ resembles magnetic anomalies observed for low-dimensional systems [60,61]. As an example, Fig. 6(a) presents magnetic specific heat simulated for a system of AFM dimers with $S=1 / 2$ assuming that exchange coupling inside the dimers $J / k_{\mathrm{B}} \approx 5.7 \mathrm{~K}$ and $75 \%$ of the magnetic Ce ions are involved in the short-range order. Close similarity between the calculated curve and the magnetic specific heat hints at a dominance of an AFM coupling between local moments of nearest neighboring $\mathrm{Ce}^{3+}$ ions. On the other hand, a layered crystal structure of $\mathrm{Ce}_{2} \mathrm{Rh}_{3} \mathrm{Sn}_{5}$ composed of $\mathrm{B}$ blocks with trivalent $\mathrm{Ce} 1$ ions separated by $\mathrm{A}$ fragments containing nonmagnetic $\mathrm{Ce} 2$ ions (see Sec. III A) may facilitate quasi-two-dimensional magnetic correlations. Further studies including neutron diffraction and inelastic scattering measurements are needed to inspect in detail the low-temperature magnetic order and magnetic excitations in $\mathrm{Ce}_{2} \mathrm{Rh}_{3} \mathrm{Sn}_{5}$.

\section{F. Electrical resistivity}

Figure 7 shows the temperature dependence of the electrical resistivity measured on three polycrystalline blocks of $\mathrm{Ce}_{2} \mathrm{Rh}_{3} \mathrm{Sn}_{5}$. The overall shape of the $\rho(T)$ curves in conjunction with the values of the resistivity of $\sim 200-300 \mu \Omega \mathrm{cm}$ at lowest temperatures indicate a metallic behavior of $\mathrm{Ce}_{2} \mathrm{Rh}_{3} \mathrm{Sn}_{5}$, as expected based on the thermodynamic and spectroscopic data, and in agreement with previous report [28].

For all the investigated specimens, the electrical resistivity shows broad maxima at $T \sim 100 \mathrm{~K}$. At similar temperatures the XAS study revealed the fastest changes in Ce valence (see Sec. III B) and the $\chi_{\mathrm{Ce} 2}(T)$ shows maximum due to excitations from Kondo singlet ground state to $j=5 / 2$ multiplet (see Sec. IIID). Therefore we explain the strong scattering in this temperature range as due to both charge and spin fluctuations associated with intermediate-valent $\mathrm{Ce}$ ions. Similar pronounced contributions to $\rho(T)$ were observed for many Ce-based IV systems $[6,43]$.

At lower temperatures, the $\rho(T)$ increases with decreasing temperature in a logarithmic fashion, as indicated in the inset of Fig. 7. Such logarithmic upturns signify the dominance of

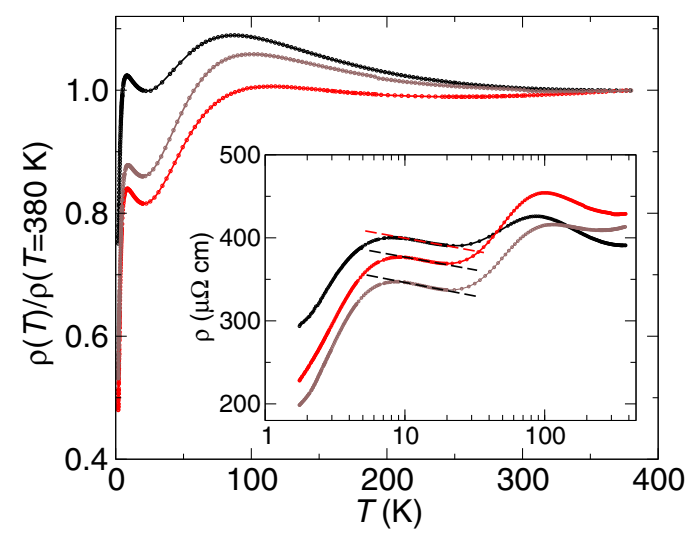

FIG. 7. The electrical resistivity of $\mathrm{Ce}_{2} \mathrm{Rh}_{3} \mathrm{Sn}_{5}$ measured on three different polycrystalline blocks normalized to the value of the resistivity at $380 \mathrm{~K}$. The inset shows the $\rho(T)$ data plotted as a function of temperature on a logarithmic scale. 
the $-\ln T$ term resulting from incoherent Kondo scattering of conduction electrons on magnetic Ce ions.

Finally, the $\rho(T)$ curves pass through maxima at temperatures of $8 \mathrm{~K}$ and fall rapidly with decreasing temperature. Such a temperature dependence of the resistivity is a typical manifestation of an onset of coherence between Kondo scattering centres arranged periodically in a crystal lattice [62]. The development of coherence governs the $\rho(T)$ at temperatures close to the magnetic transitions, which may explain the lack of evidence for the magnetic transitions in the electrical resistivity curves.

\section{G. Fixed spin moment calculations}

To get insight into the magnetic properties of $\mathrm{Ce}_{2} \mathrm{Rh}_{3} \mathrm{Sn}_{5}$ from first principles, we performed a series of electronic band structure calculations within the LSDA approximation using the fixed spin moment (FSM) method [63]. This computational technique allows to fix the total magnetic spin moment of a system and adds this constraint to the DFT treatment.

Figure 8 shows the total energies computed for different values of the FSM assuming the experimental crystal structure of $\mathrm{Ce}_{2} \mathrm{Rh}_{3} \mathrm{Sn}_{5}$. The calculations indicate that the nonmagnetic state has the lowest total energy, but there is a flat region in the total energy-vs-FSM curve ranging from 0 to about $0.4 \mu_{\mathrm{B}} /$ f.u., suggesting a proximity of a magnetic state.

Since calculated magnetic properties are often sensitive to atomic coordinates, we performed their computational

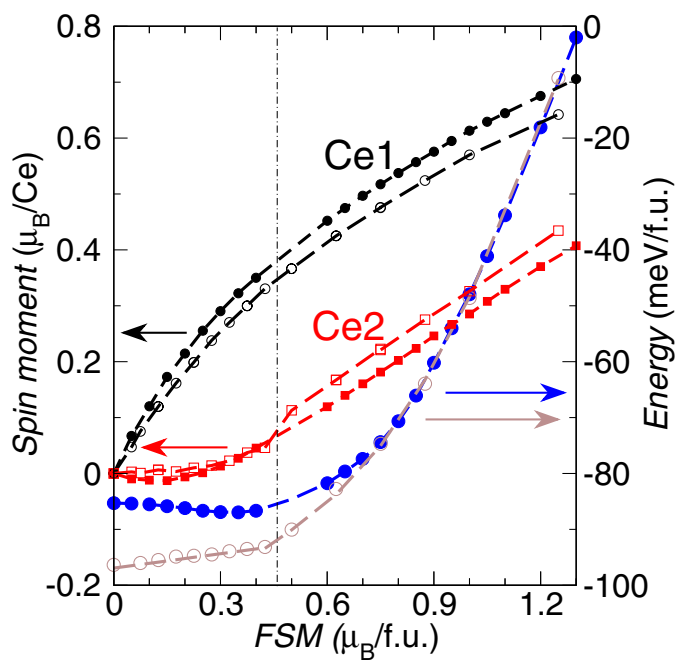

FIG. 8. The total energy-vs-FSM curves derived from FSM calculations for $\mathrm{Ce}_{2} \mathrm{Rh}_{3} \mathrm{Sn}_{5}$ within the LSDA approximation assuming either the experimental crystal structure (empty beige circles) or the LDA crystal lattice (filled blue circles). The FSM values represent the total magnetic spin moments per formula unit. The total energy scale was shifted to allow for direct comparison of the curves obtained for the experimental and LDA crystal structures. The calculated values of $\mathrm{Ce} 1$ and $\mathrm{Ce} 2$ spin moments are shown as black circles and red squares, respectively. For the experimental crystal structure, the symbols are empty, whereas for the LDA crystal lattice they are filled. The dashed lines are guided to the eye. The thin vertical dashed-dotted line separates FSM solutions with negligible spin polarization on $\mathrm{Ce} 2$ sites (on the left side) from those with $\mathrm{Ce} 2$ spin moment of at least $0.1 \mu_{\mathrm{B}}$ (on the right side). relaxation based on atomic forces [64] to find theoretical equilibrium atomic positions for the LDA approximation. The resulting internal coordinates for which total force on each atom is smaller than $5 \mathrm{mRy} / \mathrm{a}$.u. are listed in Table II. For this relaxed crystal structure, the LSDA calculations with initial spin-polarization converged to a magnetic state, in agreement with the results of thermodynamic measurements (see Secs. III D and III E). FSM calculations show that there is a shallow magnetic minimum in the energy versus FSM curve at the FSM value of $0.35 \mu_{\mathrm{B}} /$ f.u., that has now slightly lower total energy than the nonmagnetic solution $(\Delta E \approx 1.7 \mathrm{meV} / \mathrm{f}$.u; see Fig. 8). Remarkably, the magnetic moment of $0.35 \mu_{\mathrm{B}} /$ f.u. is carried basically only by $\mathrm{Ce} 1$ atoms, whereas $\mathrm{Ce} 2$ atoms as well as all $\mathrm{Rh}$ and $\mathrm{Sn}$ atoms in $\mathrm{Ce}_{2} \mathrm{Rh}_{3} \mathrm{Sn}_{5}$ stay almost unpolarized (spin moments below $0.05 \mu_{\mathrm{B}}$ per atom).

Obviously, the detailed shape of the calculated energy versus FSM curve should depend not only on the atomic positional parameters but also on the lattice parameters. Furthermore, the symmetry lowering required by various possible spin arrangements may also affect slightly the calculated total energy versus FSM curves. Nevertheless, the performed calculations for the experimental and the relaxed crystal structures indicate there is a notable energy cost associated with inducing spin polarization on $\mathrm{Ce} 2$ sites in $\mathrm{Ce}_{2} \mathrm{Rh}_{3} \mathrm{Sn}_{5}$ (Fig. 8). The minimum difference in total energy between FSM calculations giving magnetic spin moment for $\mathrm{Ce} 2$ below $\sim 0.05 \mu_{\mathrm{B}}\left(\mathrm{FSM}<0.45 \mu_{\mathrm{B}} /\right.$ f.u.; region on the left side of the vertical line on Fig. 8) and calculations resulting in spin polarization on $\mathrm{Ce} 2$ sites of at least $0.1 \mu_{\mathrm{B}}$ per $\mathrm{Ce} 2$ (region on the right side of the vertical line on Fig. 8) is of $3.5 \mathrm{meV} /$ f.u. Furthermore, for FSM calculations resulting in magnetic spin moment for $\mathrm{Ce} 2$ of $0.1 \mu_{\mathrm{B}}$, the calculated moments on $\mathrm{Ce} 1$ sites are of $0.35-0.45 \mu_{\mathrm{B}}$. Therefore we conclude that $\mathrm{Ce} 1$ ions should bear magnetic moments and contribute to the low-temperature magnetic ordering in $\mathrm{Ce}_{2} \mathrm{Rh}_{3} \mathrm{Sn}_{5}$, whereas the $\mathrm{Ce} 2$ ions should remain nonmagnetic.

\section{H. Valence band}

Figure 9 shows the total and partial atomic resolved DOSs for $\mathrm{Ce}_{2} \mathrm{Rh}_{3} \mathrm{Sn}_{5}$ calculated for the experimental crystal structure within the LDA approximation and using the LSDA $+U$ approach to account for the strong Coulomb interaction within Ce $4 f$ shell. The valence band of $\mathrm{Ce}_{2} \mathrm{Rh}_{3} \mathrm{Sn}_{5}$ consists of two parts separated by the gap of $1.5 \mathrm{eV}$. The structure at binding energies ranging from 5 to $8 \mathrm{eV}$ originates primarily from $5 s$ states of Sn atoms. The main part of the valence DOS is dominated by hybridized $\mathrm{Rh} 4 d$ and $\mathrm{Sn} 5 p$ states.

Within the LDA approximation, the Ce $4 f$ states of both $\mathrm{Ce} 1$ and $\mathrm{Ce} 2$ form narrow bands with the center of gravity above the Fermi level [see Fig. 9(a)]. The calculated number of $4 f$ electrons for $\mathrm{Ce} 1$ ions equals 1.01. Thus the electronic configuration of $\mathrm{Ce} 1$ is very close to $4 f^{1}$ expected for $\mathrm{Ce}^{3+}$. For $\mathrm{Ce} 2$ ions, however, the number of occupied $4 f$ states is of 0.92 . Such a reduced occupation of the Ce2 $4 f$ shell suggests a small deviation from the trivalent configuration, in line with a valence slightly larger than $3+$ revealed by our XAS measurements (see Sec. III B).

Since the LDA method underestimates the Coulomb repulsion of electrons within narrow bands, we performed also 


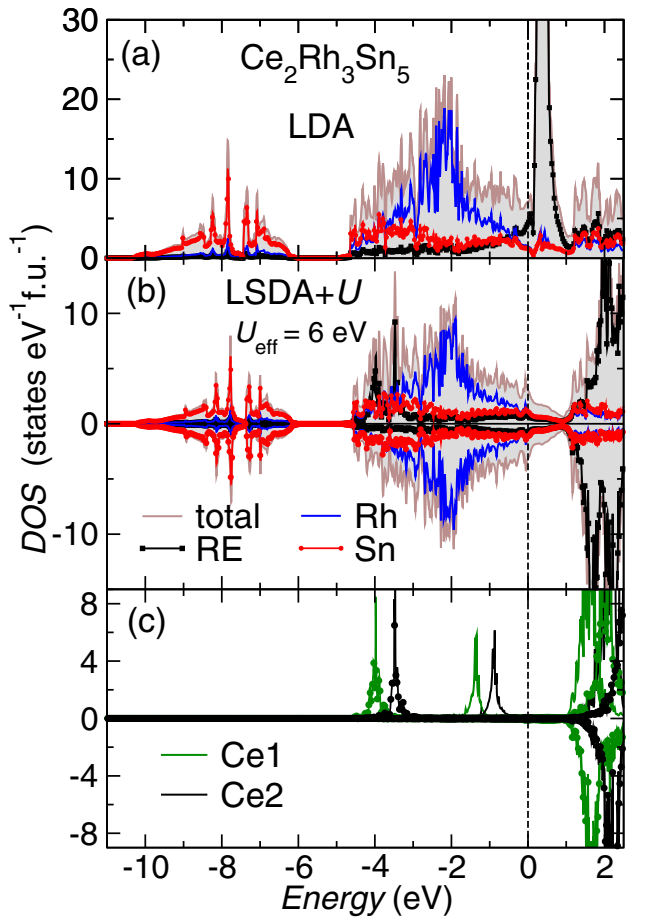

FIG. 9. The total and atomic resolved DOSs for $\mathrm{Ce}_{2} \mathrm{Rh}_{3} \mathrm{Sn}_{5}$ calculated within the LDA approximation (a) and using the LSDA $+U$ approach for the Ce $4 f$ shell [(b) and (c)] with $U_{\text {eff }}=6 \mathrm{eV} \mathrm{[65]} \mathrm{[(b);}$ thin lines with dots in (c)] or $U_{\text {eff }}=3 \mathrm{eV}$ [(thick solid lines in (c)].

additional band structure calculations using the LSDA $+U$ approach to account for stronger Coulomb interaction within Ce $4 f$ shell. Inclusion of the Hubbard-like interaction terms to the XC potential results in a shift of the occupied Ce $4 f$ bands toward higher binding energies and of the unoccupied $4 f$ states above the Fermi level for Ce ions in both lattice sites. As an example, Fig. 9(c) shows the partial DOSs for $\mathrm{Ce} 1$ and $\mathrm{Ce} 2$ calculated using the LSDA $+U$ method assuming $U_{\text {eff }}=3$ or $6 \mathrm{eV}$.

Our calculations show that for $\mathrm{Ce} 1$ the application of the $U_{\text {eff }}$ parameter larger than $\sim 2 \mathrm{eV}$ yields the qualitatively correct physical picture of $\mathrm{Ce}^{3+}$ with magnetic spin moments of $1 \mu_{\mathrm{B}} / \mathrm{Ce}$ and the occupied $4 f$ states forming narrow bands well below the $E_{\mathrm{F}}$. In case of $\mathrm{Ce} 2$, however, somewhat larger values of the $U_{\text {eff }}$ of at least $3 \mathrm{eV}$ were required to suppresses the hybridization of the $\mathrm{Ce} 24 f$ and valence-band states near the Fermi level and to move the $4 f$ bands away from the Fermi energy. We note that for $U_{\text {eff }} \gtrsim 3 \mathrm{eV}$, the binding energies of the occupied $4 f$ states are smaller by about $0.5 \mathrm{eV}$ for $\mathrm{Ce} 2$ than for Ce1, independent of the exact value of the $U_{\text {eff }}$ parameter [see Fig. 9(c)]. Furthermore, the changes in the Ce2 $4 f$ bands induced by application of the LSDA $+U$ approach lead to a narrowing of the main part of the valence band and result in a shift of $4 d$ states of $\mathrm{Rh} 2$ and $\mathrm{Rh} 3$ towards lower binding energies, whereas the strength of correlation effects within the $4 f$ shell of $\mathrm{Ce} 1$ ions has almost no impact on the other valence-band states (not shown). Thus the computational study gives an indirect evidence for the importance of the hybridization between $\mathrm{Ce} 24 f$ and conduction-band states in $\mathrm{Ce}_{2} \mathrm{Rh}_{3} \mathrm{Sn}_{5}$.

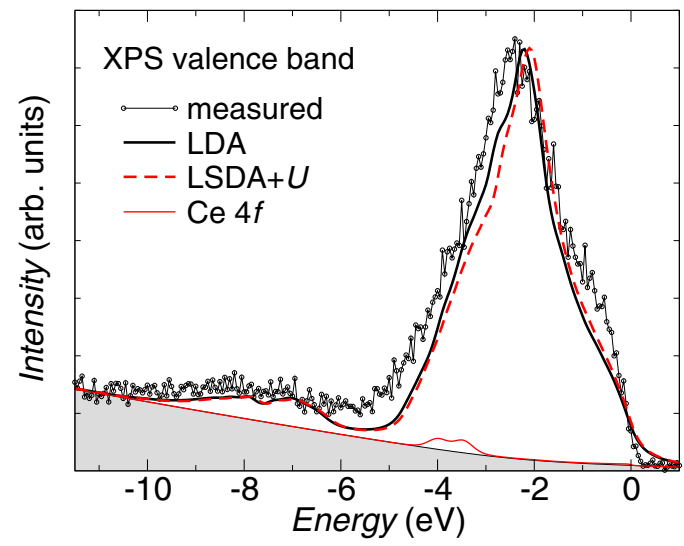

FIG. 10. XPS valence-band spectrum of $\mathrm{Ce}_{2} \mathrm{Rh}_{3} \mathrm{Sn}_{5}$ (thin black solid line with dots) in comparison with theoretical spectra calculated based on partial DOSs obtained within the LDA approximation (thick black line) and using the LSDA $+U\left(U_{\text {eff }}=6 \mathrm{eV}\right)$ [65] approach for the $\mathrm{Ce} 4 f$ shell (thick dashed red line). The thin red (grey) solid line shows the sum of partial Ce1 $4 f$ and Ce2 $4 f$ contributions. The grey field represents background estimated using the Tougaard algorithm [50].

To shed light on the effective mass enhancement in $\mathrm{Ce}_{2} \mathrm{Rh}_{3} \mathrm{Sn}_{5}$, we estimate bare values of the Sommerfeld coefficient $\gamma_{b}=\left(\pi^{2} / 3\right) k_{\mathrm{B}} \operatorname{DOS}\left(E_{\mathrm{F}}\right)$ using the $\operatorname{DOS}\left(E_{\mathrm{F}}\right)$ derived from our calculations of 6-7 st. $\mathrm{eV}^{-1}$ f.u. ${ }^{-1}$ (Fig. 9). The obtained $\gamma_{b}$ values of $15-17 \mathrm{~mJ} \mathrm{~mol}^{-1} \mathrm{~K}^{-2}$ are about seven times smaller than the experimental Sommerfeld coefficient in the paramagnetic region of $110 \mathrm{~mJ} \mathrm{~mol}^{-1} \mathrm{~K}^{-2}$ (see Sec. IIIE). Different effects such as phonon-electron coupling or low-lying magnetic excitations can enlarge the bare $\gamma_{b}$ value. However, such a strong enhancement of the Sommerfeld coefficient suggests that there is a notable renormalization of the effective quasiparticle masses due to dynamic electron-electron correlations. Since the applied computational methods (LDA and LSDA $+U$ ) are static meanfield approximations, by definition they neglect all dynamic correlation effects such as renormalization of electronic bands and the formation of Abrikosov-Suhl resonance. Therefore we conclude that none of our calculations gives a proper description of the electronic structure of $\mathrm{Ce}_{2} \mathrm{Rh}_{3} \mathrm{Sn}_{5}$ in a region close to the Fermi level. Further theoretical study based on dynamical mean-field theory (DMFT) is needed to inspect in detail the shape of the DOS in the vicinity of the Fermi energy for $\mathrm{Ce}_{2} \mathrm{Rh}_{3} \mathrm{Sn}_{5}$.

In order to gain experimental insight into the valence band of $\mathrm{Ce}_{2} \mathrm{Rh}_{3} \mathrm{Sn}_{5}$, we performed photoemission measurements. Figure 10 shows the XPS valence-band spectrum for $\mathrm{Ce}_{2} \mathrm{Rh}_{3} \mathrm{Sn}_{5}$. To facilitate its comparison with the band structure results, the theoretical XPS spectra were estimated based on the calculated partial DOSs as described in Sec. II B. The commonly used Tougaard-type [50] background (grey field in Fig. 10) was added to the calculated data to account for the presence of secondary electrons during photoemission processes. The exemplary results obtained based on DOSs calculated within the LDA approximation and using the $\mathrm{LSDA}+U\left(U_{\text {eff }}=6 \mathrm{eV}\right)$ approach are presented in Fig. 10.

The calculated curves reflect all the essential features present in the measured XPS valence-band spectrum, 
including the energy gap between the Sn $4 s$-like shallow core states and the remainder of the valence band. Thus the experiment confirms the reliability of our computational results. Although the LDA approximation seems to give a slightly better match regarding energy positions of the main features in the XPS valence-band spectrum than the LSDA $+U$ approach, the limited energy resolution of the performed XPS experiment prevents more detailed comparisons. Further, the Ce $4 f$ contributions to the XPS valence-band spectrum are very small as compared to the contributions from the other valence-band states (Fig. 10). Therefore the performed experiment also cannot give direct information about the localization of the $\mathrm{Ce} 4 f$ states in the conduction band of $\mathrm{Ce}_{2} \mathrm{Rh}_{3} \mathrm{Sn}_{5}$.

\section{CONCLUSIONS AND SUMMARY}

The crystal structure of $\mathrm{Ce}_{2} \mathrm{Rh}_{3} \mathrm{Sn}_{5}$ was refined from single crystal XRD data. Analysis of interatomic distances and coordination of $\mathrm{Ce}$ atoms occupying two distinct crystallographic sites suggests that $\mathrm{Ce} 1$ species are in a trivalent state $\left(4 f^{1}\right)$, whereas the valence of $\mathrm{Ce} 2$ ions should be larger than +3 due to extraordinarily short $\mathrm{Ce} 2-\mathrm{Rh}$ contacts. Electronic band structure calculations provide further indication for a trivalent state of $\mathrm{Ce} 1$ ions. By contrast, for $\mathrm{Ce} 2$ ions a reduced occupation of $4 f$ states to $\sim 0.92$ suggests a small deviation from the trivalent electronic configuration. Importantly, XAS measurements revealed an IV behavior. Assuming that $\mathrm{Ce} 1$ atoms are trivalent in the entire investigated temperature range, the valence of $\mathrm{Ce} 2$ species derived from the deconvolution of Ce $L_{\mathrm{III}} \mathrm{XAS}$ spectra varies between +3.18 at $6 \mathrm{~K}$ and +3.08 at $\sim 300 \mathrm{~K}$.

Thermodynamic measurements revealed a magnetic ground state for $\mathrm{Ce}_{2} \mathrm{Rh}_{3} \mathrm{Sn}_{5}$. Both specific heat and magnetization data show two magnetic transitions at $T_{\mathrm{N} 1} \approx 2.9 \mathrm{~K}$ and $T_{\mathrm{N} 2} \approx 2.4 \mathrm{~K}$. The low-temperature magnetic entropy of $\mathrm{R} \ln 2$ per formula unit implies, however, that only half of $\mathrm{Ce}$ ions in $\mathrm{Ce}_{2} \mathrm{Rh}_{3} \mathrm{Sn}_{5}$ participates in the magnetic ordering. Electronic band structure calculations indicate that only $\mathrm{Ce} 1$ ions exhibit spin polarization in $\mathrm{Ce}_{2} \mathrm{Rh}_{3} \mathrm{Sn}_{5}$. For $\mathrm{Ce} 2$ ions, the computational results in conjunction with the XPS spectra point to the importance of the hybridization of the $4 f$ and conduction-band states. This hybridization is crucial for the formation of a nonmagnetic Kondo-singlet state characterized by an enhanced Pauli-like magnetic susceptibility $\chi(0) \approx 0.003 \mathrm{emu} / \mathrm{mol}$ and a large electronic specific heat $\gamma \approx 110 \mathrm{~mJ} \mathrm{~mol}^{-1} \mathrm{~K}^{-2}$, which is about seven times larger than the bare $\gamma_{b}$ value derived from the calculated single-particle $\operatorname{DOS}\left(E_{\mathrm{F}}\right)$. Such a strong augmentation of the Sommerfeld coefficient supports the important role of electron-electron correlation effects, whereas the Sommerfeld-Wilson ratio $R_{\mathrm{SW}} \approx 1$ provides a further indication that both $\chi(0)$ and $\gamma$ are similarly enhanced due to heavy quasiparticles in the FL state.

With increasing temperature, the strong Pauli-like behavior in the magnetic susceptibility is going over to a local moment paramagnetism. Finally, near room temperature $\mathrm{Ce}_{2} \mathrm{Rh}_{3} \mathrm{Sn}_{5}$ behaves as an ordinary paramagnet with nearly full moment expected for all the $\mathrm{Ce}$ ions being in a trivalent state and a small positive $\chi_{0-\mathrm{HT}} \approx 8 \times 10^{-5} \mathrm{emu} / \mathrm{mol}$ which, after correcting for core level diamagnetism and Landau diamagnetism, is comparable to the Pauli susceptibility expected based on the $\operatorname{DOS}\left(E_{\mathrm{F}}\right)$ derived from our electronic structure calculations.

To summarize, our combined experimental and theoretical study indicates that $\mathrm{Ce}_{2} \mathrm{Rh}_{3} \mathrm{Sn}_{5}$ is a Kondo lattice system in which at low temperatures a magnetic order due to trivalent $\mathrm{Ce} 1$ ions coexists with an intermediate-valence behavior of $\mathrm{Ce} 2$ ions with a nonmagnetic Kondo-singlet ground state. Further study is needed to elucidate in detail the electronic band structure of $\mathrm{Ce}_{2} \mathrm{Rh}_{3} \mathrm{Sn}_{5}$ near the Fermi level where dynamical many-body effects determine the shape of quasiparticle DOS and to shed more light on the magnetic ground state.

\section{ACKNOWLEDGMENTS}

The authors thank Christoph Geibel from Max-Planck Institute for Chemical Physics of Solids for fruitful discussions. The authors are grateful to Jerzy Kubacki from University of Silesia for his kind help with XPS measurements and to E. Welter and D. Zajac from HASYLAB for their helpful assistance during XAS measurements on beamline C of Hasylab, Desy. M. G. would like to acknowledge financial support from the Max-Planck Society and the DAAD foundation through research fellowships.
[1] G. Stewart, Rev. Mod. Phys. 73, 797 (2001); A. Georges, G. Kotliar, W. Krauth, and M. J. Rozenberg, ibid. 68, 13 (1996), and references therein.

[2] A. C. Hewson, The Kondo Problem to Heavy Fermions (Cambridge University Press, Cambridge, UK, 1997).

[3] H. B. Radousky (ed.), Magnetism in Heavy Fermion Systems, (World Scientific, Singapore, 2000).

[4] P. Monthoux, D. Pines, and G. G. Lonzarich, Nature (London) 450, 1177 (2007).

[5] G. R. Stewart, Rev. Mod. Phys. 78, 743 (2006).

[6] J. M. Lawrence, P. S. Riseboroughs, and R. D. Parks, Rep. Prog. Phys. 44, 1 (1981), and references therein.

[7] M. A. Ruderman and C. Kittel, Phys. Rev. 96, 99 (1954).
[8] S. Doniach, in Valence Instability and Related Narrow Band Phenomena, edited by R. D. Parks (Plenum, New York, 1977), and references therein.

[9] T. Mishra, W. Hermes, U. Ch. Rodewald, R.-D. Hoffmann, and R. Pöttgen, Z. Anorg. Allg. Chem. 634, 470 (2008).

[10] V. Eyert, E.-W. Scheidt, W. Scherer, W. Hermes, and R. Pöttgen, Phys. Rev. B 78, 214420 (2008).

[11] L. Durivault, F. Bourée, B. Chevalier, G. André, J. Etourneau, and O. Isnard, J. Magn. Magn. Mater. 232, 139 (2001).

[12] F. Tappe, W. Hermes, M. Eul, and R. Pöttgen, Intermetallics 17, 1035 (2009).

[13] S. Linsinger, M. Eul, W. Hermes, R.-D. Hoffmann, and R. Pöttgen, Z. Naturforsch. 64b, 1345 (2009). 
[14] S. F. Matar, J. F. Riecken, B. Chevalier, R. Pöttgen, A. F. Al Alam, and V. Eyert, Phys. Rev. B 76, 174434 (2007).

[15] J. A. Mydosh, A. M. Strydom, M. Baenitz, B. Chevalier, W. Hermes, and R. Pöttgen, Phys. Rev. B 83, 054411 (2011).

[16] R. Feyerherm, E. Dudzik, K. Prokeš, J. A. Mydosh, Y.-K. Huang, and R. Pöttgen, Phys. Rev. B 90, 041104 (2014).

[17] J. M. Lawrence, M. F. Hundley, J. D. Thompson, G. H. Kwei, and Z. Fisk, Phys. Rev. B 43, 11057 (1991).

[18] O. Trovarelli, J. G. Sereni, G. Schmerber, and J. P. Kappler, Physica B 206-207, 243 (1995).

[19] K. Ghosh, S. Ramakrishnan, S. K. Dhar, S. K. Malik, G. Chandra, V. K. Pecharsky, K. A. Gschneidner, Jr., Z. Hu, and W. B. Yelon, Phys. Rev. B 52, 7267 (1995).

[20] M. B. Gamża, A. Ślebarski and H. Rosner, J. Phys.: Condens. Matter 20, 025201 (2008).

[21] D. Niepmann, R. Pöttgen, B. Künnen, G. Kotzyba, C. Rosenhahn, and B. D. Mosel, Chem. Mater. 11, 1597 (1999).

[22] M. B. Gamża, A. Ślebarski, and H. Rosner, Eur. Phys. J. B 63, 1 (2008).

[23] M. B. Gamża, W. Schnelle, R. Gumeniuk, Yu. Prots, A. Ślebarski, H. Rosner, and Yu. Grin, J. Phys.: Condens. Matter 21, 325601 (2009).

[24] M. B. Gamża, W. Schnelle, A. Ślebarski, U. Burkhardt, R. Gumeniuk, and H. Rosner, J. Phys.: Condens. Matter 20, 395208 (2008).

[25] M. B. Gamża et al. (unpublished).

[26] A. Ślebarski, N. A. Frederick, and M. B. Maple, Philos. Mag. B 82, 1275 (2002); A. Ślebarski, J. Spałek, M. B. Gamża, and A. Hackemer, Phys. Rev. B 73, 205115 (2006); J. Spałek, A. Slebarski, J. Goraus, L. Spałek, K. Tomala, A. Zarzycki, and A. Hackemer, ibid. 72, 155112 (2005).

[27] M. B. Gamża, A. Ślebarski, and H. Rosner, Eur. Phys. J. B 67, 483 (2009).

[28] N. G. Patil and S. Ramakrishnan, Phys. Rev. B 59, 12054 (1999).

[29] M. Méot-Mayer, G. Venturini, B. Malaman, J. Steinmetz, and B. Roques, Mater. Res. Bull. 19, 1181 (1984).

[30] See Supplemental Material at http://link.aps.org/supplemental/ 10.1103/PhysRevB.95.165142 for details of powder X-ray diffraction measurements and metallographic investigations on polycrystalline samples of $\mathrm{Ce}_{2} \mathrm{Rh}_{3} \mathrm{Sn}_{5}$.

[31] R. C. Clark and J. S. Reid, Acta Cryst. A 51, 887 (1995).

[32] P. J. Becker and P. Coppens, Acta Cryst. A 30, 129 (1974).

[33] V. Petricek, M. Dusek, and L. Palatinus, Z. Kristallogr. 229, 345 (2014).

[34] B. Ravel and M. Newville, J. Synchrotron Rad. 12, 537 (2005).

[35] Y. Baer, G. Bush, and P. Cohn, Rev. Sci. Instrum. 46, 466 (1975).

[36] K. Koepernik and H. Eschrig, Phys. Rev. B 59, 1743 (1999).

[37] J. P. Perdew and Y. Wang, Phys. Rev. B 45, 13244 (1992).

[38] H. Eschrig, K. Koepernik, and I. Chaplygin, J. Solid State Chem. 176, 482 (2003).

[39] J. J. Yeh and J. Lindau, At. Data Nucl. Data Tables 32, 1 (1985).

[40] R. V. Skolozdra, in Handbook on the Physics and Chemistry of Rare Earth, edited by K. A. Gschneider, Jr. and L. Eyring (North-Holland, Amsterdam, 1997), Vol. 24, p. 400.

[41] J. Emsley, The Elements (Oxford University Press, New York, 1998).
[42] J. C. Fuggle, F. U. Hillebrecht, Z. Zolnierek, R. Lässer, Ch. Freiburg, O. Gunnarsson, and K. Schönhammer, Phys. Rev. B 27, 7330 (1983); A. J. Signorelli and R. G. Hayes, ibid. 8, 81 (1973).

[43] D. Kaczorowski, A. P. Pikul, U. Burkhardt, M. Schmidt, A. Ślebarski, A. Szajek, M. Werwiński, and Yu. Grin, J. Phys.: Condens. Matter 22, 215601 (2010).

[44] R. Gumeniuk, R. Sarkar, C. Geibel, W. Schnelle, C. Paulmann, M. Baenitz, A. A. Tsirlin, V. Guritanu, J. Sichelschmidt, Yu. Grin, and A. Leithe-Jasper, Phys. Rev. B 86, 235138 (2012); R. Sarkar, R. Gumeniuk, A. Leithe-Jasper, W. Schnelle, Yu. Grin, C. Geibel, and M. Baenitz, ibid. 88, 201101 (2013).

[45] I. Abbati, L. Braicovich, B. Michelis, A. Fasana, G. L. Olcese, F. Canepa, and G. A. Costa, Solid State Commun. 55, 1081 (1985).

[46] E. V. Sampathkumaran, Hyperfine Interact. 27, 183 (1986).

[47] Chandan Mazumdar, R. Nagarajan, C. Godart, L. C. Gupta, B. D. Padalia, and R. Vijayaraghavan, J. Appl. Phys. 79, 6347 (1996).

[48] U. B. Paramanik, Anupama, U. Burkhardt, R. Prasad, C. Geibel, and Z. Hossain, J. Alloy. Comput. 580, 435 (2013).

[49] S. Doniach and M. Šunjić, J. Phys. C 3, 285 (1970).

[50] S. Tougaard and P. Sigmund, Phys. Rev. B 25, 4452 (1982).

[51] O. Gunnarsson and K. Schönhammer, Phys. Rev. B 28, 4315 (1983).

[52] P. W. Selwood, Magnetochemistry (Interscience, New York, 1956).

[53] S. M. M. Evans, T. Chung, and G. A. Gehring, J. Phys.: Condens. Matter 1, 10473 (1989).

[54] V. T. Rajan, Phys. Rev. Lett. 51, 308 (1983).

[55] N. E. Bickers, D. L. Cox, and J. W. Wilkins, J. Magn. Magn. Mater. 47-48, 335 (1985), and references therein.

[56] A. C. Hewson and J. W. Rasul, J. Phys. C: Solid State Phys. 16, 6799 (1983).

[57] P. Fulde, Routes to Heavy Fermions, in Magnetism in Metals: A Symposium in Memory of Allan Mackintosh, edited by D. F. McMorrow, J. Jensen, and H. M. Ronnow (Det Kongelige Danske Videnskabernes Selskab, Denmark, 1997), Vol. 45, p. 165.

[58] H. Yashima, H. Mori, N. Sato, and T. Satoh, J. Magn. Magn. Mater. 31-34, 411 (1983).

[59] K. D. Schotte and U. Schotte, Phys. Lett. A 55, 38 (1975).

[60] D. C. Mattis, The Theory of Magnetism (Harper and Row, New York, 1965).

[61] A. Orendáčová, M. Kajňaková, J. Černák, J.-H. Park, E. Čižmár, M. Orendáč, A. Vlček, O. V. Kravchyna, A. G. Anders, A. Feher, and M. W. Meisel, Chem. Phys. 309, 115 (2005).

[62] D. L. Cox and N. Greve, Z. Phys. B: Condens. Matter 71, 321 (1988).

[63] K. Schwarz and P. Mohn, J. Phys. F 14, L129 (1984).

[64] R. Yu, D. Singh, and H. Krakauer, Phys. Rev. B 43, 6411 (1991).

[65] Y. Baer, H. R. Ott, J. C. Fuggle, and L. E. De Long, Phys. Rev. B 24, 5384 (1981); J. K. Lang, Y. Baer, and P. A. Cox, J. Phys. F 11, 121 (1981); J. F. Herbst, R. E. Watson, and J. W. Wilkins, Phys. Rev. B 17, 3089 (1978); J. F. Herbst and J. W. Wilkins, Phys. Rev. Lett. 43, 1760 (1979); V. I. Anisimov and O. Gunnarsson, Phys. Rev. B 43, 7570 (1991), and references therein. 